\title{
Spatial Reuse and Fairness of Ad Hoc Networks With Channel-Aware CSMA Protocols
}

\author{
Yuchul Kim, François Baccelli, and Gustavo de Veciana, Fellow, IEEE
}

\begin{abstract}
We investigate the benefits of channel-aware (opportunistic) scheduling of transmissions in ad hoc networks. The key challenge in optimizing the performance of such systems is finding a good compromise among three interdependent quantities: 1) the density of scheduled transmitters; 2) the quality of transmissions; and 3 ) the long term fairness among nodes. We propose two new channel-aware slotted CSMA protocols opportunistic CSMA and quantile-based CSMA (QT-CSMA) and develop new stochastic geometric models to quantify their performance in terms of spatial reuse and spatial fairness. When properly optimized, these protocols offer substantial improvements in performance relative to CSMA-particularly, when the density of nodes is moderate to high. In addition, we show that a simple version of QT-CSMA can achieve robust performance gains without requiring careful parameter optimization. The quantitative results in this paper suggest that channel-aware scheduling in ad hoc networks can provide substantial benefits which might far outweigh the associated implementation overheads.
\end{abstract}

Index Terms-Ad hoc networks, ALOHA, CSMA, O-CSMA, opportunistic scheduling, quantile scheduling, QT-CSMA, spatial fairness, spatial reuse.

\section{INTRODUCTION}

$\mathbf{E}$ VALUATING and optimizing the capacity of wireless ad hoc networks has been one of the goals of the networking and information theory research communities over the last decade. Due to the inherent randomness in such networks, e.g., locations of nodes, wireless channels, and node interactions governed by protocols, researchers have developed stochastic models that can parsimoniously capture the uncertainty of such environments while still giving insight on system performance and optimization. Work based on stochastic geometric models has perhaps been the most successful in terms of providing reasonably realistic, yet mathematically tractable, results; see [1]-[3]. This paper leverages this line of work to study the performance of networks operated under two channel-aware slotted CSMA type protocols.

Manuscript received June 6, 2011; revised June 5, 2013; accepted December 2, 2013. This work was supported in part by the National Science Foundation under Grant CNS-0917067 and in part by the Texas Advanced Computing Center, University of Texas at Austin. G. de Veciana was supported by the Intel 5G Research Program. This paper was presented at the 2011 Spatial Stochastic Models for Wireless Networks Workshop.

Y. Kim and G. de Veciana are with the Department of Electrical and Computer Engineering, University of Texas at Austin, Austin, TX 78712 USA (e-mail: yuchul.kim@gmail.com; gustavo@ece.utexas.edu).

F. Baccelli is with the Department of Computer Science, INRIA ENS, Paris 75214, France (e-mail: francois.baccelli@ens.fr).

Communicated by R. D. Yates, Associate Editor for Communication Networks.

Color versions of one or more of the figures in this paper are available online at http://ieeexplore.ieee.org.

Digital Object Identifier 10.1109/TIT.2014.2320909
One of the important factors determining the performance of a wireless network is the degree of spatial reuse. Spatial reuse is a measure quantifying the degree of spectrum reuse per unit space. In this work, we consider a random wireless network using a single frequency band where nodes are uniformly distributed in space. Every slot, each node decides whether or not to transmit to its receiver based the activity of surrounding nodes. A sparse or dense set of transmitters would have low spatial reuse or and strong interference respectively. The other important factor is spatial fairness which measures the degree of fairness in the performance of spatially distributed nodes. There will be inherent performance unfairness due to the interaction of random node locations and contention for media access. This can be mitigated by balancing the transmission opportunity and success rates across spatially distributed nodes. It is the Medium Access Control (MAC) protocol that makes transmission decisions and thus shapes the spatial reuse and fairness patterns nodes will see.

MAC protocols such as ALOHA, Opportunistic ALOHA (O-ALOHA), and CSMA have been studied in detail, so we briefly introduce and discuss them.

ALOHA is a basic MAC protocol in which spatially distributed nodes simply transmit with a probability $p$. A mathematical model for a spatial version of an ALOHA based wireless ad-hoc network is presented in [1]; various extensions capturing the impact of modulation techniques on the transmission capacity have been studied; see [2]. Because transmitters contend independently, the transmission probability $p$ should be properly chosen as a function of node density so as to achieve a high spatial reuse. This involves finding a compromise between a high density of transmitters and excessive interference which deteriorates the quality of transmissions and accordingly leads to low spatial reuse.

In [4] and [5], the performance of an opportunistic version of spatial ALOHA (O-ALOHA) ${ }^{1}$ was evaluated. In these models, only qualified transmitters, namely nodes whose channel to their associated receivers have channel gain that exceeds a threshold $\gamma$, can transmit with probability $p$. The resulting spatial reuse is thus affected by two parameters. When properly tuned, this simple channel-aware MAC can increase spatial reuse by roughly $40 \%$ relative to simple ALOHA.

Although O-ALOHA can significantly increase spatial reuse by qualifying nodes seeing good channels, it still suffers from collisions which limit its performance. Unlike ALOHA based protocols, Carrier Sense based Medium Access (CSMA)

\footnotetext{
${ }^{1}$ The ALOHA considering channel state information (a.k.a opportunistic ALOHA) in single hop network was introduced and studied in [6] and [7].
} 


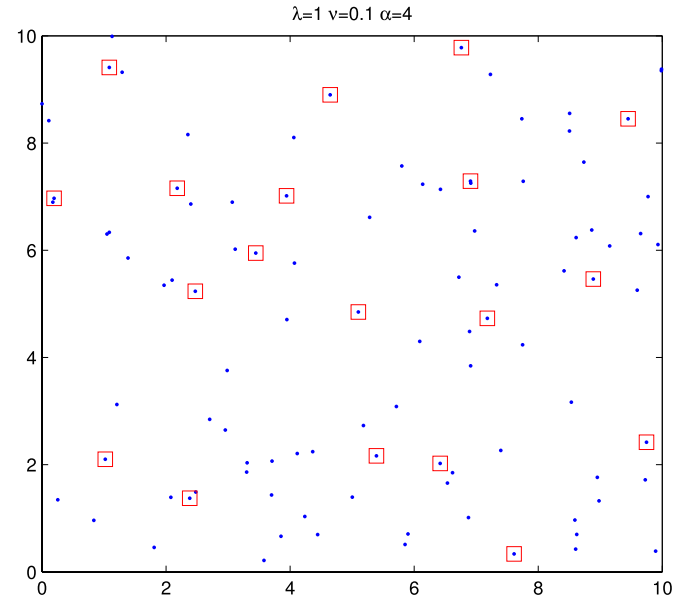

Fig. 1. A realization of modified Matérn hardcore process: randomly distributed points are the realization of marked Poisson point process where each point has an independent identically distributed mark denoting its timer value in $[0,1]$. If a point has the smallest timer value in its neighborhood (neighborhood is not shown here but formally defined later in (1)), then, it is selected as a CSMA transmitter. Selected CSMA transmitters were drawn inside boxes.

protocols achieve high spatial reuse by coordinating transmissions among neighboring nodes so as to avoid collisions. In [8] and [9], a modified Matérn hardcore process model for a spatial slotted CSMA protocol was introduced. Each node contends with its 'neighbors' via a uniformly distributed contention timer. The node with the earliest timer wins. As a result the active transmitters end up being well separated; see Fig. 1. The model suggests CSMA can increase spatial reuse by roughly $25 \%$ over basic ALOHA.

In this paper, we extend the CSMA ad-hoc network model introduced in [9] to study two simple channel-aware MAC protocols. In the first scheme, named Opportunistic CSMA (O-CSMA), we use a channel quality threshold $\gamma$, as introduced in [4] and [5], to qualify nodes which participate in the CSMA contention process. Optimizing the performance of such networks requires selecting $\gamma$ as a function of node density and channel variation distributions. In the second scheme, called QuanTile-based CSMA (QT-CSMA), nodes contend based on the quantile of the channel quality to their associated receivers. Doing so allows nodes to transmit when their channel is the 'best' in their neighborhood. This also ensures that each node gets a fair share of access opportunities among the nodes in its neighborhood, and circumvents the problem of choosing a density dependent qualification threshold. This is particularly desirable if channel statistics seen across nodes are heterogeneous. Quantile-based scheduling approaches for downlinks in cellular networks were introduced and studied in [10]-[13] and in the wireless LAN setting in [14] and [15].

The performance metrics considered in this paper are spatial averages of network performance, which means that the performance metric captures an average over possible realizations of nodes' locations. This is particularly meaningful, since in real world scenarios nodes are irregularly placed and/or motion might make a performance metric which is a function of nodes' location less informative. To that end, we characterize the performance as seen by a typical node using tools from stochastic geometry together with analytical/numerical computation methods.

\section{A. Contributions}

This paper makes the following four contributions. First, to the best of our knowledge, it presents the first attempt to evaluate CSMA-based opportunistic MAC protocols in ad-hoc networks, namely O-CSMA and QT-CSMA. Our new stochastic geometry analysis captures the delicate interactions between the channel gains and interference statistics underlying the performance of opportunistically scheduled nodes in ad-hoc networks.

Second, we evaluate the sensitivity of spatial reuse to various protocol parameters, showing the advantages of QT-CSMA over O-CSMA which in turn has substantially better performance than ALOHA based schemes. To that end, we characterize the interplay between the density of active transmitters and the quality of transmissions in the function of the qualification threshold $\gamma$ and a carrier sense threshold $\nu$.

Third, this paper is the first to evaluate the spatial fairness realized by these protocols and shows that QT-CSMA can achieve better fairness than CSMA. Specifically we introduce and quantify a spatial fairness index among sets of nodes sharing the same number of neighbors, which captures the impact of random nodes' placements.

Finally, we study tradeoffs between spatial fairness and spatial reuse, and compare the Pareto optimal performance points of O-CSMA with those of QT-CSMA. In particular, we show that quantile-based CSMA without a qualification step ( $\mathrm{QT}_{0}$-CSMA) achieves a performance comparable to that of O/QT-CSMA in terms of both fairness and density of successful transmission, it is thus a robust and attractive choice from an engineering perspective. An initial discussion of implementation considerations for such protocols can found in [16].

\section{B. Related Work}

Since the introduction of the IEEE 802.11 protocol, several researchers have attempted to analyze multi-hop wireless networks using the IEEE 802.11. [17] was one of the early efforts which provided an analytical model for a given fixed network and computed the lower bound on the sum throughput of transmitter-receiver pairs for a given network. However, the model's simplified physical layer, the so-called protocol model, did not account for the impact of aggregate interference. Later, [18] provided a more sophisticated model which takes into account various PHY and MAC layer parameters. The authors propose a linear approximation of the access probability of individual nodes as a function of its success probability and developed a linear system relationship relating the success probabilities and transmission probabilities of nodes in a given network. This gave a reasonable approximation of the per-node throughput, however, the work did not reveal how the system was affected by various system parameter selections or the inherent randomness in wireless environment. Furthermore performance was evaluated for a given fixed network, which does not provide insight regarding typical ad hoc networks. 
The above limitations - i.e., not taking into account random fading channel, random node locations, impact of aggregate interference, and capture effect ${ }^{2}$ - are naturally addressed in research based on stochastic geometric models; see [1]-[3], [19], on which our work is based. In this line of work, the performance metrics of interest are an average over random environments (including fading, node locations, protocols, etc), which can be more informative in terms of representing typical behavior. Specifically the CSMA related work of [9] and [8] used a spatial point process to model spatially distributed wireless nodes using a CSMA-like MAC protocol. These works successfully approximated the statistics of the aggregate interference resulting from CSMA-like MAC nodes by those of a non-homogeneous Poisson point process of interferers. The approximation was validated via simulation and was shown to match well. However, characterizing the exact interference statistics is still very hard and has remained an open problem. As a response to this, subsequent work in [20] and [21] suggested an alternative approximation for the performance of CSMA nodes which is accurate for asymptotically sparse networks. Models capturing carrier sense mechanism have also been successfully used to study cognitive radio networking scenarios in [22]-[24].

Our work is different from the above work in the following aspects. First, we build upon the CSMA model in [9] incorporating opportunistic CSMA scheduling schemes. We consider the dependency among the channel gain of a scheduled node, contention resolution mechanism, and the activity of the surrounding nodes (or accordingly the statistics of interference), which has to the best of our knowledge not been previously explored. We elaborate a parameterized model which is flexible enough to be used to study various protocols from ALOHA to QT-CSMA. Second, we consider the fairness for slotted (or synchronized) CSMA networks. In particular, we study how system parameters and opportunistic CSMA protocols can change fairness characteristics of the slotted CSMA network.

\section{Organization}

In Section II we describe our system model, including details for our two proposed opportunistic MAC protocols. In Section III the transmission and success probability of a typical node under the two MAC protocols are derived. These will be used later to compute the two performance metrics. In Section IV we compare the spatial reuse of O-CSMA and QT-CSMA networks, and in Section $\mathrm{V}$ the fairness of such networks is evaluated and tradeoffs between spatial reuse and fairness are considered under various parameter values. Conclusions are given in Section VI.

\section{System Model}

\section{A. Node Distribution and Channel Model}

We model an ad-hoc wireless network as a set of transmitters and their corresponding receivers. Transmitters are

\footnotetext{
${ }^{2}$ If two transmitters happen to send their packets to the same receiver, the one with a higher signal strength can be received with non-zero probability. This is called the capture effect.
}

randomly distributed on $\mathbb{R}^{2}$ as a marked homogeneous Poisson Point Process (PPP) $\Psi=\left\{X_{i}, E_{i}, T_{i}, \mathbf{F}_{i}, \mathbf{F}_{i}^{\prime}\right\}$, where $\Phi \equiv\left\{X_{i}\right\}_{i \geq 1}$ is the PPP with density $\lambda$ denoting the set of transmitters' locations and $E_{i}$ is an indicator function which is equal to 1 if a node $X_{i}$ transmits and 0 otherwise. The value of $E_{i}$ is determined by the MAC protocol used and the activity of other nodes $\left\{X_{j}\right\}_{j \neq i}$. $T_{i}$ denotes the timer value used by node $X_{i}$ for contention resolution with its neighboring nodes. Node $X_{i}$ transmits if it has the smallest timer value in its neighborhood. The value of $T_{i}$ is determined by the timer selection algorithm. We assume that the distance between a transmitter and its associated receiver is $r$. The direction from a transmitter to its receiver is randomly and uniformly distributed on $[0,2 \pi]$. Throughout this paper, we only consider the performance as seen by a typical receiver.

Let $\mathbf{F}_{i}=\left(F_{i j}: j\right)$ be a vector of random variables $F_{i j}$ denoting the fading channel gain between the $i$ th transmitter and the receiver associated with the $j$ th transmitter. In this work, we consider i.i.d. block fading channel model, in which a fading gain is independently sampled for the duration of each time slot with an identical distribution. Note that $F_{i i}$ denotes the channel gain from the $i$ th transmitter to its associated receiver. We assume that the random variables $F_{i j}$ are identically distributed (i.i.d.) with mean $\mu^{-1}$, i.e., $F_{i j} \sim F$, with cumulative distribution function (cdf) $G(x)=P(F \leq x)$. Let $\mathbf{F}_{i}^{\prime}=\left(F_{i j}^{\prime}: j\right)$ be the vector of random variables $F_{i j}^{\prime}$ denoting the fading gain between the $i$-th transmitter and the $j$-th transmitter. These are assumed to be symmetric ${ }^{3}$ and i.i.d, i.e., $F_{i j}^{\prime}=F_{j i}^{\prime}$ and $F_{i j}^{\prime} \sim F$. In this paper, we only consider the Rayleigh fading case where $F$ has an exponential distribution with $G(x)=1-\exp (-\mu x)$ for $x \geq 0$, but other fading models could be considered. Let $\|x\|$ denote the norm of $x \in \mathbb{R}^{2}$ and $\|x-y\|^{\alpha}$ be the path loss between two locations $x \in \mathbb{R}^{2}$ and $y \in \mathbb{R}^{2}$ where the pathloss exponent $\alpha>2$. Then, the interference power that the $j$-th receiver at location $y$ experiences from the $i$-th transmitter at location $x$ is then given by $F_{i j}\|x-y\|^{-\alpha}$.

\section{B. Signal to Interference and Noise Ratio Model}

The performance of a receiver is governed by its signal to interference plus noise ratio (SINR). Under the model given above, the SINR seen at the $i$-th receiver is

$$
\operatorname{SINR}_{i}=\frac{F_{i i} r^{-\alpha}}{I_{\Phi \backslash\left\{X_{i}\right\}}+W}
$$

where $I_{\Phi \backslash\left\{X_{i}\right\}}=\sum_{j: X_{j} \in \Phi \backslash\left\{X_{i}\right\}} E_{j} F_{j i}\left\|Y_{i}-X_{j}\right\|^{-\alpha}$ is the aggregate interference power ${ }^{4}$, or so-called shot noise, and $W$ is the thermal noise power. We shall focus on interference limited networks, where the impact of thermal noise is comparatively negligible, so let $W=0$. The reception model we consider is the so-called outage reception model, where a receiver can successfully decode a transmission if its received SINR exceeds a decoding threshold $t$.

\footnotetext{
${ }^{3}$ Unlike $F_{i j}^{\prime}, F_{i j}$ is not symmetric, i.e., $F_{i j} \neq F_{j i}$.

${ }^{4} Y_{i}$ is the receiver associated with transmitter $X_{i}$.
} 


\section{Carrier Sense Multiple Access Protocols}

We consider a slotted CSMA network, where nodes compete with each other to access a shared medium. Carrier sensing is followed by data transmission at each slot. Each node contends with its 'neighboring' nodes using a (uniformly distributed on $[0,1])$ timer value. The timer value is independent of everything else and each node transmits if it has the smallest timer value in its neighborhood and defers otherwise. CSMA provides a way to resolve contentions among nodes but does not take advantage of channel variations. In what follows, we introduce two distributed opportunistic CSMA protocols which take advantage of channel variations amongst transmitters and their receivers: opportunistic CSMA (O-CSMA) and Quantile-based CSMA (QT-CSMA).

Under O-CSMA, nodes whose channel gains are higher than a fixed threshold $\gamma$ qualify to contend; we call this the qualification process. We assume that channel quality $F_{i i}$ is available to transmitter $X_{i}$ at each slot. Qualified nodes in turn, contend for transmission with their neighbors on that slot. Specifically, let $\Phi^{\gamma}=\left\{X_{i} \in \Phi \mid F_{i i}>\gamma\right\}$ denote the set of qualified nodes or contenders. Note that $\Phi^{\gamma}$ is a subset of $\Phi$ which is generated by independent marks with probability

$$
p_{\gamma}=\mathbb{P}(F>\gamma),
$$

so it is a homogeneous PPP with density $\lambda^{\gamma} \equiv \lambda p_{\gamma}$. Each contender $X_{i} \in \Phi^{\gamma}$ has a set of qualified nodes with which it contends. We say two transmitters $X_{i}$ and $X_{j}$ contend if the received interference they see from each other is larger than the carrier sense threshold $v$, i.e., if $F_{i j}^{\prime}\left\|X_{i}-X_{j}\right\|^{-\alpha}>v$ and by symmetry $F_{j i}^{\prime}\left\|X_{i}-X_{j}\right\|^{-\alpha}>v$. We call the set of contenders for a qualified node $i$ its neighborhood and denote it by

$$
\mathcal{N}_{i}^{\gamma}=\left\{X_{j} \in \Phi^{\gamma} \text { s.t. } F_{j i}^{\prime}\left\|X_{i}-X_{j}\right\|^{-\alpha}>v, j \neq i\right\} .
$$

Contending nodes are not allowed to transmit simultaneously since they can potentially interfere with each other. To avoid collisions, in every slot each node $X_{j}$ in $\Phi^{\gamma}$ picks a random timer value $T_{j}$ which is uniformly distributed on $[0,1]$. At the start of each time slot, node $X_{j}$ starts its own timer which expires in $T_{j}$. Each node senses the medium until its own timer expires. If no node (in its neighborhood) begins transmitting prior to that time, then, it starts transmitting, otherwise it defers. Under this mechanism, a node $X_{i}$ transmits only if the node's timer value is the minimum in its neighborhood, i.e., when $T_{i}$ is equal to $\min _{j: X_{j} \in \mathcal{N}_{i}^{\gamma} \cup\left\{X_{i}\right\}} T_{j}$. Note that this timer value based carrier sense model could be easily extended to incorporate RTS-CTS based carrier sense mechanism but to simplify we will not consider this here.

Note that the qualification process is a mechanism for selecting nodes with high channel gains. (all qualified nodes have channel gains larger than $\gamma$.) The posterior channel distribution after qualification is that of $F$ given that $F>\gamma$, so it is given by a shifted exponential distribution

$$
G_{\gamma}(x) \equiv \mathbb{P}(F<x \mid F>\gamma)=\left(1-\exp ^{-\mu(x-\gamma)}\right) \mathbf{1}\{x \geq \gamma\} .
$$

The qualification process not only increases the signal strength but also reduces the number of interferers, so we can expect more successful transmissions. However, the parameter $\gamma$ should be chosen judiciously; otherwise there will either be too many transmitting nodes generating too much interference or too few transmitting nodes resulting in low spatial reuse. Neither case is desirable. Note that when $\gamma=0$ this model corresponds to the standard CSMA model analyzed in [9].

Under QT-CSMA there is also a qualification process with threshold $\gamma$. However, the active transmitters in a neighborhood are selected based on the quantile of their current channel gain; we refer to this as quantile scheduling. Specifically, we assume that channel quality $F_{i i}$ is available to transmitter $X_{i}$, and at each slot a qualified transmitter $X_{i}$ computes its channel quantile ${ }^{5} Q_{i}=G_{\gamma}\left(F_{i i}\right)$ using the distribution for the channel gain $F_{i i}$ conditioned on $F_{i i}>\gamma$. This transforms the channel distribution to a uniform distribution on $[0,1]$, which serves both as a relative indicator of channel quality and to determine the timer for collision avoidance. More precisely, under QT-CSMA, $X_{i}$ sets its timer value $T_{i}$ to $1-Q_{i}$ and senses the medium until its timer expires. If no transmitting node is detected prior $T_{i}$, then, the node accesses the medium, otherwise it defers. In other words, node $X_{i}$ transmits only if it has the highest quantile in its neighborhood, i.e., if $Q_{i}=Q_{i}^{\max }$ where $Q_{i}^{\max } \equiv \max _{j: X_{j} \in \mathcal{N}_{i}^{\gamma} \cup\left\{X_{i}\right\}} Q_{j}$. Let $F_{i, \gamma}^{\max }=G_{\gamma}^{-1}\left(Q_{i}^{\max }\right)$ be the channel fade of a transmitting node $X_{i}$ or the channel fade given node $X_{i}$ transmits, where $G_{\gamma}^{-1}(\cdot)$ is the inverse function of $G_{\gamma}(\cdot)$. Let $N_{i}^{\gamma}=\left|\mathcal{N}_{i}^{\gamma}\right|$; then $F_{i, \gamma}^{\max }$ is a $N_{i}^{\gamma}+1$ th order statistic, i.e.,

$$
F_{i, \gamma}^{\max }=\max \left[F_{1, \gamma}, F_{2, \gamma}, \ldots, F_{N_{i}^{\gamma}+1, \gamma}\right],
$$

with the random variables $F_{j, \gamma}$ i.i.d. The distribution of $F_{i, \gamma}^{\max }$ conditioned on $N_{i}^{\gamma}=n$ is given by

$$
\mathbb{P}\left(F_{i, \gamma}^{\max } \leq x \mid N_{i}^{\gamma}=n\right)=\left(1-\exp ^{-\mu(x-\gamma)}\right)^{n+1} \mathbf{1}\{x \geq \gamma\} .
$$

Thus QT-CSMA further exploits opportunism beyond the qualification process. Unlike O-CSMA, a QT-CSMA node transmits only when it has the best channel condition in its neighborhood, which should further improve its likelihood of successful transmission. One may surmise that QT-CSMA may work well even without the qualification phase since quantile scheduling will fully take advantage of opportunistic node selection gain (so-called multi-user diversity). This will be explored later. We shall denote QT-CSMA with $\gamma=0$ by $\mathrm{QT}_{0}$-CSMA.

\section{Notation}

For a positive random variable $I$, let $\mathcal{L}_{I}(s)=\mathbb{E}\left[e^{-s I}\right]$ be the Laplace transform of $I$. Given a countable set $\mathcal{C}$, let $|\mathcal{C}|$ be the cardinality of $\mathcal{C}$. Let $\mathbf{1}\{\cdot\}$ denote the indicator function and let $B_{l} \equiv b(0, l)$ denote a ball centered at the origin with radius $l$. $\mathbb{R}_{+}$denotes the set of non-negative real numbers. Let $\Phi$ be a stationary point process and $\mathcal{Y}$ be a property of $\Phi$. We let $\mathbb{P}^{0 !}$ denote the reduced Palm probability of $\Phi$. Intuitively, the probability that $\Phi$ satisfies the property $\mathcal{Y}$ under $\mathbb{P}^{0 !}$ is the conditional probability that $\Phi \backslash\{0\}$ satisfies property $\mathcal{Y}$

\footnotetext{
${ }^{5}$ In practice, this requires the knowledge of $G_{\gamma}$.
} 
TABLE I

SUMMARY OF NOTATIONS

\begin{tabular}{c|l}
\hline$X_{i}$ & $i$-th transmitter or its location in $\mathbb{R}^{2}$ \\
$Y_{i}$ & $i$-th receiver associated with $X_{i}$ or its location in $\mathbb{R}^{2}$ \\
$\Phi$ & Poisson point process denoting the set of transmitters $\left\{X_{i}\right\}_{i \geq 1}$ \\
$\lambda$ & Density of nodes in $\Phi$ \\
$F$ & Generic exponential random variable with mean $1 / \mu$ denoting fading gain \\
$F_{i j}$ & 20cmFading gain between transmitter $X_{i}$ and the receiver $Y_{j}$ \\
$F_{i j}^{\prime}\left(=F_{j i}^{\prime}\right)$ & Fading gain between transmitter $X_{i}$ and transmitter $X_{j}$ \\
$\gamma$ & Qualification threshold \\
$p_{\gamma}$ & Probability that a transmitter is qualified $(=\mathbb{P}(F>\gamma))$ \\
$\Phi^{\gamma}$ & Set of qualified transmitters \\
$F_{\gamma}$ & Generic random variable denoting posterior channel distribution $\mathbb{P}(F<x \mid F>\gamma)$ \\
$G_{\gamma}(\cdot)$ & Cdf of random variable $F_{\gamma}$ \\
$\Phi_{M}^{\gamma}$ & Set of active transmitters using CSMA protocol \\
$\Phi_{M}^{\gamma 0}$ & Set of active transmitters $\Phi_{M}^{\gamma}$ given $0 \in \Phi_{M}^{\gamma}$ \\
$\nu$ & Carrier sense threshold \\
$t$ & Decoding threshold \\
$\lambda^{\gamma}$ & Density of qualified transmitters $\left(=\lambda p_{\gamma}\right)$ \\
$N_{0}^{\gamma}$ & Size of neighborhood of a typical node $X_{0}$ \\
$F_{0, \gamma}^{\text {max }}\left(N_{0}^{\gamma}\right)$ & Fading gain of a typical QT-CSMA transmitter when the size of its neighborhood is $N_{0}^{\gamma}$ \\
$r$ & Distance between a transmitter and its associated receiver \\
$N_{s, 0}^{\gamma}$ & Size of neighborhood of a typical node under the assumption $F_{i j}^{\prime}=\mathbb{E}[F]$ in Section V-A \\
$I_{\Phi_{M}^{\gamma 0}}$ & Aggregate interference power from transmitters in $\Phi_{M}^{\gamma 0 !}$ \\
$I_{\Phi_{M}^{n}, x}^{\gamma 0 !}$ & Aggregate interference power from transmitters in $\Phi_{M}^{\gamma 0 !}$ conditioned on that the associated \\
$\lambda_{d e n s}$ & typical node has $N_{0}^{\gamma}=n$ contenders and it has channel gain $F_{0, \gamma}^{\text {max }}\left(N_{0}^{\gamma}\right)=x$ \\
$\Phi_{M}^{d e n s}$ & Asymptotic density of active transmitters \\
$p_{t x}$ & Point process of active CSMA transmitters with density $\lambda_{\text {dens }}$ \\
$p_{s u c}$ & Access probability of a typical transmitter \\
$I_{\Phi}$ & Transmission success probability of a typical receiver of an active link \\
\hline & Aggregate interference power from transmitters in $\Phi$ \\
\hline
\end{tabular}

given that $\Phi$ has a point at 0 . This will be denoted as follows: $\mathbb{P}(\Phi \backslash\{0\} \in \mathcal{Y} \mid 0 \in \Phi)=\mathbb{P}^{0}(\Phi \backslash\{0\} \in \mathcal{Y})=\mathbb{P}^{0 !}(\Phi \in \mathcal{Y})$. We define $\Phi^{0}$ as a point process $\Phi$ given $0 \in \Phi$ and we define $\Phi^{0 \text { ! }}$ as a point process with the distribution of $\Phi^{0} \backslash\{0\}$, i.e., $\Phi^{0 !} \equiv$ $\Phi^{0} \backslash\{0\} . \mathbb{E}^{0}$ denotes Palm expectation, which is interpreted as the conditional expectation conditioned on a node at the origin; see [19] and [25] for detailed definitions. For convenience a summary of notation discussed so far, and introduced in the sequel is provided in Table I.

\section{TRANSMISSION PERFORMANCE ANALYSIS}

In this section, we derive expressions for the access and transmission success probabilities which in turn are used to compute the density of successful transmissions for our opportunistic scheduling schemes. We begin by defining performance metrics and restating some suitable modified results from [4], [9] to fit our setting.

\section{A. Spatial Reuse}

As a measure of spatial reuse, we will use the density of successful transmissions which is defined as the mean number of nodes that successfully transmit per square meter. This is given by

$$
d_{s u c}=\lambda p_{t x} p_{s u c}
$$

where $\lambda$ denotes the density of transmitters, $p_{t x}$ denotes the transmission probability of a typical transmitter, and $p_{s u c}$ denotes the transmission success probability ${ }^{6}$. This metric not only measures the level of spatial packing through $\lambda p_{t x}$ but also the quality of transmissions through $p_{s u c}$, which captures the interactions (though interference) among spatially distributed nodes.

\section{B. Previous Results}

Below we briefly introduce several key results that we will use in the sequel.

Proposition 1 (Laplace Transform of Shot-Noise for Non-homogeneous Poisson Field-[9, Sec. 18.5.2]): Let $\Phi_{h}=$ $\left\{X_{i}, F_{i}\right\}$ be an independently marked non-homogeneous PPP in $\mathbb{R}^{2}$ with spatial density $h(x)$. Then, the Laplace transform of the associated shot-noise interference

$$
I_{\Phi_{h}}(w)=\sum_{i:\left(X_{i}, F_{i}\right) \in \Phi_{h}} F_{i}\left\|X_{i}-w\right\|^{-\alpha}
$$

at location $w \in \mathbb{R}^{2}$ is given by

$$
\begin{aligned}
\mathcal{L}_{I_{\Phi_{h}}(w)}(s) & =\mathbb{E}\left[e^{-s I_{\Phi_{h}}(w)}\right] \\
& =\exp \left\{-\int_{\mathbb{R}^{2}} 1-\mathcal{L}_{F}\left(\frac{s}{\|x-w\|^{\alpha}}\right) h(x) d x\right\} .
\end{aligned}
$$

\footnotetext{
${ }^{6}$ Note that in this work $p_{t x}$ corresponds to the fraction of active transmitters in a slot and $p_{s u c}$ is the fraction of receivers successfully receiving data given that their corresponding transmitters are active in the slot. $d_{s u c}$ gives the density of transmitter and receiver pairs which transmit and receive in the slot. This type of snapshot analysis does not require random variables to have a time index.
} 
In particular, if $F_{i} \sim F$ is an exponential random variable with rate $\mu$, we have

$$
\mathcal{L}_{I_{\Phi_{h}}(w)}(s)=\exp \left\{-\int_{\mathbb{R}^{2}} \frac{h(x)}{1+\frac{\mu}{s}\|x-w\|^{\alpha}} d x\right\} .
$$

Proposition 2 (Mean Neighborhood Size-[9, Sec. 18.3]): The number of neighbors of a typical node under the model in Section II is Poisson with mean

$$
\begin{aligned}
\bar{N}_{0}^{\gamma} & =\mathbb{E}\left[N_{0}^{\gamma}\right] \\
& =\mathbb{E}^{0 !}\left[\sum_{i: X_{i} \in \Phi^{\gamma}} \mathbf{1}\left\{F_{i i}>v\left\|X_{i}\right\|^{\alpha}\right\}\right] \\
& =\lambda^{\gamma} \int_{\mathbb{R}^{2}} \exp \left\{-v \mu\|x\|^{\alpha}\right\} d x \\
& =\frac{2 \pi \lambda^{\gamma} \Gamma(2 / \alpha)}{\alpha(v \mu)^{2 / \alpha}} .
\end{aligned}
$$

Proposition 3 (Conditional Transmission Probability Under CSMA Protocol-[9, Corollary 18.4.3]): For the O-CSMA model given in Section II with qualified transmitter density $\lambda^{\gamma}$, the probability that a qualified node $x_{1} \in \mathbb{R}^{2}$ transmits given there is a transmitter $x_{0} \in \mathbb{R}^{2}$ with $\left\|x_{1}-x_{0}\right\|=\tau$ which transmits (i.e., wins its contention), i.e., $P\left(E_{1}=1 \mid E_{0}=1\right.$, $\left.\left\{x_{0}, x_{1}\right\} \subset \Phi^{\gamma},\left\|x_{1}-x_{0}\right\|=\tau\right) \equiv h\left(\tau, \lambda^{\gamma}\right)$, is

$h\left(\tau, \lambda^{\gamma}\right)=\frac{\frac{2}{b\left(\tau, \lambda^{\gamma}\right)-\bar{N}_{0}^{\gamma}}\left(\frac{1-e^{-\bar{N}_{0}^{\gamma}}}{\bar{N}_{0}^{\gamma}}-\frac{\left.1-e^{-b(\tau, \lambda \gamma}\right)}{b\left(\tau, \lambda^{\gamma}\right)}\right)\left(1-e^{-\nu \mu \tau^{\alpha}}\right)}{\frac{1-e^{-\bar{N}_{0}^{\gamma}}}{\bar{N}_{0}^{\gamma}}-e^{-v \mu \tau^{\alpha}}\left(\frac{1-e^{-\bar{N}_{0}^{\gamma}}}{\left(\bar{N}_{0}^{\gamma}\right)^{2}}-\frac{e^{-\bar{N}_{0}^{\gamma}}}{\bar{N}_{0}^{\gamma}}\right)}$,

where

$$
\begin{aligned}
& b\left(\tau, \lambda^{\gamma}\right) \\
& \quad=2 \bar{N}_{0}^{\gamma}-\lambda^{\gamma} \int_{0}^{\infty} \int_{0}^{2 \pi} e^{-v \mu\left(x^{\alpha}+\left(\tau^{2}+x^{2}-2 \tau x \cos \theta\right)^{\frac{\alpha}{2}}\right)} x d \theta d x .
\end{aligned}
$$

The function $h(\cdot)$, shown in the Fig. 2a as blue solid curve, denotes the density of non-homogenous Poisson point process at a location $\tau$ away from an active transmitter. Unlike the density of a homogenous PPP which is just a constant, that of the interferers has very low density around the origin and constant density for large $\tau$. This non-homogeneous density captures the impact of the carrier sense mechanism and controlled interference in CSMA networks. This is an exact result and it will be used below to build the best Poisson approximation for active CSMA transmitters. In Section III-D.2, we further develop the two-fold measure to capture its dependency on the channel gain and the number of neighboring nodes.

\section{O-CSMA}

1) Access Probability of a Typical Transmitter: The access probability is the probability that a typical node transmits. As described earlier, under O-CSMA, only nodes who qualify can contend, so the network after the qualification process is indeed equivalent to a CSMA network with node density $\lambda^{\gamma}$. The channel distribution function of a qualified node, say $X_{i}$ is given by (2). Let $E_{i}=\mathbf{1}\left\{F_{i i}>\gamma, T_{i}<\min _{j: X_{j} \in \mathcal{N}_{i}^{\gamma}} T_{j}\right\}$ be the

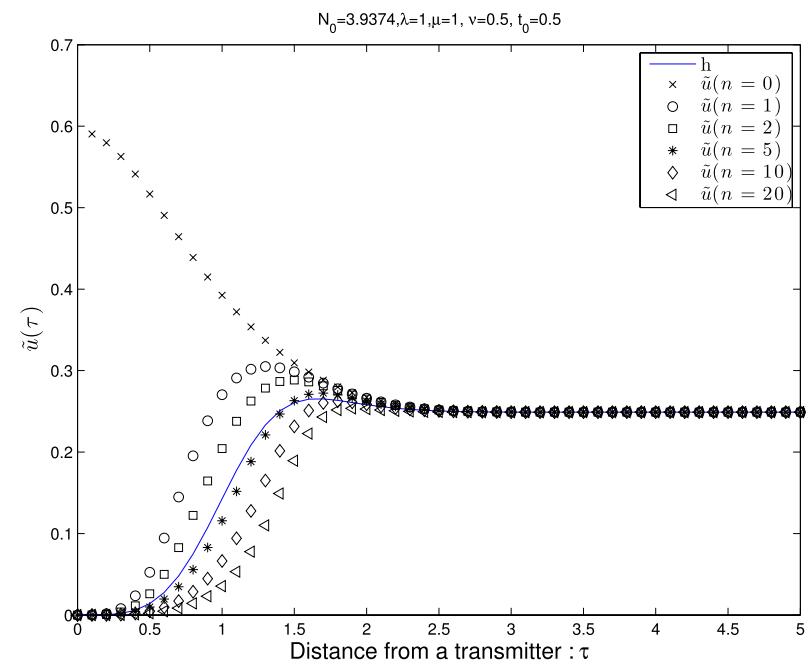

(a)

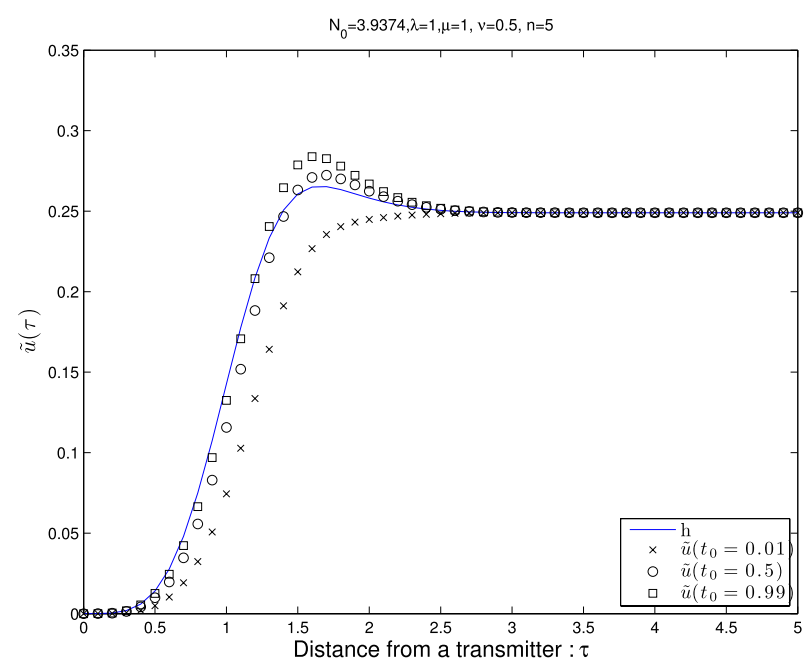

(b)

Fig. 2. $h(\cdot)$ in blue line is a function computed in (8). $\tilde{u}(\cdot)$ is a function we computed above for $N_{0}=n \geq 0$ and $t_{0} \in[0,1)$. (a) $\tilde{u}(\tau)$ is shown for various number of $n$. If $n$ is larger, i.e., many transmitters are seen by the center node $y_{0}$, then node $y_{1}$ will also see many nodes and accordingly have low transmission probability. (b) $\tilde{u}(\tau)$ is shown for various number of $t_{0}$. If $t_{0}$ is large, $y_{0}$ is will lose in contention with high probability. Accordingly $y_{1}$ will have high transmission probability.

transmission indicator for $X_{i} \in \Phi$, i.e., which indicates if $X_{i}$ qualifies and wins the contention process in its neighborhood, and $\Phi_{M}^{\gamma}=\left\{X_{i} \in \Phi \mid E_{i}=1\right\}$ be the set of active transmitters. We define the transmission probability of the typical node (which is at the origin) as

$$
p_{t x}^{o p}(\lambda, \gamma, \nu)=\mathbb{P}^{0}\left(F_{00}>\gamma, T_{0}<\min _{j: X_{j} \in \mathcal{N}_{0}^{\gamma}} T_{j}\right) .
$$

Note that the two events in (10) are independent. To compute the probability of the second event, we condition on $T_{0}$, i.e.,

$$
\mathbb{P}^{0}\left(T_{0}<\min _{j: X_{j} \in \mathcal{N}_{0}^{\gamma}} T_{j}\right)=\mathbb{E}_{T_{0}}^{0}\left[\mathbb{P}^{0}\left(T_{0}<\min _{j: X_{j} \in \mathcal{N}_{0}^{\gamma}} T_{j} \mid T_{0}\right)\right] .
$$

The conditional probability in the above expectation is the probability that $X_{0}$ has no neighboring node whose timer value 
is less than $T_{0}$, i.e.,

$$
\begin{gathered}
\mathbb{P}^{0 !}\left(\left\{X_{j} \in \Phi \text { s.t. } F_{j j}>\gamma, F_{j 0}^{\prime}>v\left\|X_{j}-X_{0}\right\|^{\alpha},\right.\right. \\
\left.\left.j \neq 0, \quad T_{j}<T_{0}\right\}=\emptyset \mid T_{0}\right) .
\end{gathered}
$$

The density measure of such nodes at location $x \in \mathbb{R}^{2}$ with $F_{j j}=f_{1}, F_{j 0}=f_{2}$ and $T_{j}=m$ is

$$
\begin{aligned}
\Lambda\left(d m, d f_{1}, d f_{2}, d x\right)= & \mathbf{1}\left\{m<T_{0}\right\} d m \mathbf{1}\left\{f_{1}>\gamma\right\} G\left(d f_{1}\right) \\
& \times \mathbf{1}\left\{f_{2}>v\|x\|^{\alpha}\right\} G\left(d f_{2}\right) \lambda d x .
\end{aligned}
$$

Thus, the conditional void probability of such nodes, i.e., (12), is

$$
\begin{aligned}
\exp \left\{-\int_{\mathbf{R}^{2}}\right. & \left.\int_{0}^{\infty} \int_{0}^{\infty} \int_{0}^{1} \Lambda\left(d m, d f_{1}, d f_{2}, d x\right)\right\} \\
& =\exp \left\{-T_{0} p_{\gamma} \int_{\mathbb{R}^{2}} 1-G\left(v\|x\|^{\alpha}\right) \lambda d x\right\} \\
& =\exp \left\{-T_{0} p_{\gamma} \bar{N}_{0}\right\} .
\end{aligned}
$$

Substituting (14) into (11) gives

$$
p_{t x}^{o p}(\lambda, \gamma, \nu)=\frac{1-\exp \left\{-p_{\gamma} \bar{N}_{0}\right\}}{\bar{N}_{0}} .
$$

Note that the spatial mean number of contenders for a typical node under O-CSMA is given by $p_{\gamma} \bar{N}_{0}$ since individual nodes qualify with probability $p_{\gamma}$. The case with $\gamma=0$ (or $p_{\gamma}=1$ ) corresponds to the pure CSMA scheme without a qualification step.

2) Transmission Success Probability of a Typical Receiver: Next, we compute the transmission success probability of a receiver associated with a typical active transmitter. This is equivalent to the success probability of the receiver of transmitter $X_{0}=0$ given $X_{0} \in \Phi_{M}^{\gamma}$ :

$$
p_{\text {suc }}^{o p}(\lambda, \gamma, \nu, t)=\mathbb{P}^{0 !}\left(\frac{F_{00} r^{-\alpha}}{I_{\Phi_{M}^{\gamma}}^{\gamma}}>t \mid F_{00}>\gamma\right),
$$

where

$$
I_{\Phi_{M}^{\gamma}} \equiv \sum_{j: X_{j} \in \Phi_{M}^{\gamma}} F_{j 0}\left\|X_{j}-(0, r)\right\|^{-\alpha}
$$

is the interference seen at the receiver at $(0, r)^{7}$. Note that in (15) we could replace $\mathbb{P}^{0 !}$ and $I_{\Phi_{M}^{\gamma}}$ by $\mathbb{P}^{0}$ and $I_{\Phi_{M}^{\gamma} \backslash\{0\}}$.

We shall denote the shot noise as seen by the receiver a distance $r$ away from an active transmitter at the origin by $I_{\Phi_{M}^{\gamma}}$. When we refer to the shot noise outside $\mathbb{P}^{0}(\cdot)$, we will use $I_{\Phi_{M}^{\gamma 0}}$ instead of $I_{\Phi_{M}^{\gamma}}$ to explicitly denote that $X_{0} \in \Phi_{M}^{\gamma 0}$. Then, the shot noise of interest can be written as

$$
I_{\Phi_{M}^{\gamma 0 !}} \equiv \sum_{j: X_{j} \in \Phi_{M}^{\gamma 0 !}} F_{j 0}\left\|X_{j}-(0, r)\right\|^{-\alpha} .
$$

For notational simplicity let $F_{\gamma}$ be a random variable with the distribution function (2) which is independent of $F_{00}$. Then, (15) can be rewritten as follows by conditioning on $F_{\gamma}$ :

$$
\mathbb{P}^{0 !}\left(F_{\gamma}>\operatorname{tr}^{\alpha} I_{\Phi_{M}^{\gamma}}\right)=\mathbb{E}_{F_{\gamma}}\left[\mathbb{P}^{0 !}\left(F_{\gamma}>t r^{\alpha} I_{\Phi_{M}^{\gamma}} \mid F_{\gamma}\right)\right] .
$$

\footnotetext{
${ }^{7}$ Due to the symmetry of PPP, we can simply assume that the receiver at distance $r$ from its transmitter is placed at $(0, r)$.
}

Note that it is hard to compute (18) since $\Phi_{M}^{\gamma 0 !}$ is a point process induced by the qualification process followed by the CSMA protocol, which has dependency among node locations. It is called the Matern CSMA process [9]. Thus, following [9], we approximate the shot noise $I_{\Phi_{M}^{\gamma 0 !}}$ with $I_{\Phi_{h}^{\gamma 0 !}}=$ $\sum_{j: X_{j} \in \Phi_{h}^{\gamma 0 !}} F_{j 0}\left\|X_{j}-(0, r)\right\|^{-\alpha}$ which is a shot noise seen at the receiver of $X_{0}$ in a non-homogeneous PPP $\Phi_{h}^{\gamma}$ with density $\lambda^{\gamma} h\left(\tau, \lambda^{\gamma}\right)$ for $\tau>0$, where $\lambda^{\gamma} \equiv p_{\gamma} \lambda$ and $h(\tau, \lambda)$ is the conditional probability that a CSMA transmitter at distance $\tau$ from the origin be active conditioned on an active CSMA transmitter at the origin with the density of nodes being $\lambda^{\gamma}$; see (8). Since $h$ is a function which converges to 0 as $\tau \rightarrow 0$, and converges to $p_{t x}^{o p}$ as $\tau \rightarrow \infty$, it captures well the modification of the interference due to the presence of the transmitter at the origin. The $h$ function (blue solid curve) is shown in Fig. 2a for certain parameter set. Using this approach we have that

$$
\mathbb{P}^{0 !}\left(F_{\gamma}>t r^{\alpha} I_{\Phi_{M}^{\gamma}}\right) \approx \mathbb{E}_{F_{\gamma}}\left[\mathbb{P}^{0 !}\left(F_{\gamma}>\operatorname{tr}^{\alpha} I_{\Phi_{h}^{\gamma}} \mid F_{\gamma}\right)\right] .
$$

Let $\xi_{h}(x)$ be the probability density function of $I_{\Phi_{h}^{\gamma 0} \backslash\{0\}}$, then, using an indicator function, we can rewrite the right hand side of (19) as

$$
\mathbb{E}_{F_{\gamma}}\left[\int_{-\infty}^{\infty} \xi_{h}(x) \mathbf{1}\left\{0<x<\frac{F_{\gamma}}{t r^{\alpha}}\right\} d x\right] .
$$

Clearly $\mathbf{1}\left\{0<x<\frac{F_{\gamma}}{t r^{\alpha}}\right\}$ is square integrable for $r>0$ and $t>0$, and $\xi_{h}(x)$ is also square integrable ${ }^{8}$. Then by applying the Plancherel-Parseval Theorem in [26, Ch. 3.3, p.157] to (20) followed by a change of variables, (20) becomes

$$
\int_{-\infty}^{\infty} \mathcal{L}_{\Phi_{\Phi_{h}^{\gamma 0 !}}}\left(2 i \pi t r^{\alpha} s\right) \frac{\mathcal{L}_{F_{\gamma}}(-2 i \pi s)-1}{2 \pi i s} d s .
$$

Noting that $\mathcal{L}_{F_{\gamma}}(s)=\frac{\mu}{\mu+s} e^{-s \gamma}$, we get that

$$
\begin{aligned}
& p_{\text {suc }}^{o p}(\lambda, \gamma, v, t) \\
& \quad \approx \int_{-\infty}^{\infty} \mathcal{L}_{I_{\Phi_{h}^{\gamma 0 !}}}\left(2 i \pi r^{\alpha} t s\right) \frac{\frac{\mu}{\mu-2 i \pi s} \exp \{2 i \pi s \gamma\}-1}{2 i \pi s} d s .
\end{aligned}
$$

The last step is to compute the Laplace transform $\mathcal{L}_{\Phi_{\Phi_{h}^{\gamma 0 !}}}(s)$ which is given as

$$
\mathcal{L}_{\Phi_{h}^{\gamma 0 !}}(s)=\exp \left\{-\lambda^{\gamma} \int_{0}^{\infty} \int_{0}^{2 \pi} \frac{h\left(\tau, \lambda^{\gamma}\right) \tau d \theta d \tau}{1+\mu f(\tau, r, \theta) / s}\right\}
$$

where $f(\tau, r, \theta)=\left(\tau^{2}+r^{2}-2 \tau r \cos \theta\right)^{\frac{\alpha}{2}}$. Replacing (23) into (22) gives a numerically computable integral form for the outage probability.

\section{D. $Q T-C S M A$}

1) Access Probability of a Typical Transmitter: Computing the access probability of a typical QT-CSMA node is not much different from that of an O-CSMA node. Under

\footnotetext{
${ }^{8}$ Note that the pdf of Poisson shot noise stemming from a PPP with finite density is square integrable; see [4]. The existence of a Poisson point process the shot noise of which dominates $I_{\Phi_{h}^{\gamma 0}}$ implies that the pdf of $I_{\Phi_{h}^{\gamma 0}}$ is square integrable.
} 
QT-CSMA, a node can transmit if its timer expires first or equivalently if it has the highest quantile in its neighborhood. Let $E_{i}$ be the transmission indicator of node $X_{i} \in \Phi$, i.e., $E_{i}=\mathbf{1}\left\{F_{i i}>\gamma, Q_{i}>\max _{j: X_{j} \in \mathcal{N}_{i}^{\gamma}} Q_{j}\right\}$. Let $\Phi_{M}^{\gamma}=$ $\left\{X_{i} \in \Phi\right.$ s.t. $\left.E_{i}=1\right\}$ be a thinned version of $\Phi$ containing only active transmitters. Then, using a technique similar to that used above, the access probability of a typical node $X_{0}$ at the origin under QT-CSMA is computed as follows:

$$
p_{t x}^{q t}(\lambda, \gamma, \nu)=\mathbb{E}^{0}\left[\frac{p_{\gamma}}{N_{0}^{\gamma}+1}\right]=\frac{1-\exp \left\{-p_{\gamma} \bar{N}_{0}\right\}}{\bar{N}_{0}} .
$$

Since all $Q_{i}$ s are uniform random variables, the result is the same as (14).

2) Transmission Success Probability of a Typical Receiver: Next we compute the transmission success probability of a receiver associated with a typical transmitter $X_{0}$ at the origin. To determine the success probability, we need to characterize the fading gain $F_{0, \gamma}^{\max }$ and the interference power that the receiver experiences. We shall explicitly denote the fact that $F_{0, \gamma}^{\max }$ depends on $N_{0}^{\gamma}$ by writing $F_{0, \gamma}^{\max }\left(N_{0}^{\gamma}\right)$ in what follows. The aggregate interference from concurrent active transmitters in $\Phi_{M}^{\gamma 0 !}$ to the receiver of $X_{0}$ is given by $I_{\Phi^{\gamma 0 !}}$ as in (17). Then, the success probability of a typical QT-CSMA receiver is

$$
p_{s u c}^{q t}(\lambda, \gamma, \nu, t)=\mathbb{P}^{0 !}\left(F_{0, \gamma}^{\max }\left(N_{0}^{\gamma}\right)>\operatorname{tr}^{\alpha} I_{\Phi_{M}^{\gamma}}\right) .
$$

Unlike in (18), $F_{0, \gamma}^{\max }\left(N_{0}^{\gamma}\right)$ is no longer independent of $I_{\Phi_{M}^{\gamma 0 !}}$. To see this intuitively, consider two extreme cases. First, suppose $F_{0, \gamma}^{\max }\left(N_{0}^{\gamma}\right)$ has a very small value, say $\epsilon$; then, this implies the channel gains of $X_{0}$ 's neighbors are concentrated within the small interval $[0, \epsilon]$; so, the neighbors of $X_{0}$ 's neighbors are not likely to defer their transmissions, which in turn means $X_{0}$ 's receiver would experience a somewhat stronger interference. By contrast, if $F_{0, y}^{\max }\left(N_{0}^{\gamma}\right)$ has a large value, say $\omega$, then, the fading gains of $X_{0}$ 's neighbors are distributed on $[0, \omega]$, which is more likely to cause their neighbors to defer. This on average makes the interference level seen at the receiver smaller than in the previous case.

That is, $I_{\Phi_{M}^{\gamma 0 !}}$ depends on both $N_{0}^{\gamma}$ and $F_{0, \gamma}^{\max }\left(N_{0}^{\gamma}\right)$. By conditioning on $N_{0}^{\gamma}$ and $F_{0, \gamma}^{\max }\left(N_{0}^{\gamma}\right)$, (25) can be written as

$$
\mathbb{E}^{0 !}\left[\mathbb{P}^{0 !}\left(F_{0, \gamma}^{\max }\left(N_{0}^{\gamma}\right)>\operatorname{tr}^{\alpha} I_{\Phi_{M}^{\gamma}} \mid N_{0}^{\gamma}, F_{0, \gamma}^{\max }\left(N_{0}^{\gamma}\right)\right)\right] .
$$

As in (18), we approximate $I_{\Phi_{M}^{\gamma 0}}$ for a given $N_{0}^{\gamma}=n$ and $F_{0, \gamma}^{\max }\left(N_{0}^{\gamma}\right)=x$ by a random variable $I_{\Phi_{u}^{\gamma}}$ denoting the inter- ference induced by a non-homogeneous Poisson point process $\Phi_{u}^{\gamma}$ with density $\lambda^{\gamma} u(n, x, \tau, \lambda, \gamma)$, where $u(n, x, \tau, \lambda, \gamma)$ is the conditional probability that a node $y_{1}$ transmits conditioned on the following facts: 1) $y_{0}$ transmits, i.e., $E_{0}=1,2$ ) $N_{0}^{\gamma}=n$, 3) $F_{0, \gamma}^{\max }\left(N_{0}^{\gamma}\right)=x$ or equivalently $y_{0}$ 's timer value $T_{0}$ is given by $t_{0}=1-G_{\gamma}(x)$, 4) both $y_{0}$ and $y_{1}$ belong to $\Phi^{\gamma}$, and 5) $y_{1}$ is $\tau$ away from $y_{0}$. This can be written as

$$
\begin{aligned}
u(n, x, \tau, \lambda, \gamma) & =\mathbb{P}\left(E_{1}=1 \mid E_{0}=1, N_{0}^{\gamma}=n,\right. \\
F_{0, \gamma}^{\max }\left(N_{0}^{\gamma}\right) & \left.=x,\left\{y_{0}, y_{1}\right\} \subset \Phi^{\gamma},\left\|y_{0}-y_{1}\right\|=\tau\right) .
\end{aligned}
$$

Using the fact that $1-G_{\gamma}(x)$ is a one-to-one mapping from $[\gamma, \infty]$ to $[0,1]$, we can rewrite $(27)$ as

$$
\begin{aligned}
u(n, x, \tau, \lambda, \gamma) & =\mathbb{P}\left(E_{1}=1 \mid E_{0}=1, N_{0}^{\gamma}=n,\right. \\
T_{0} & \left.=t_{0}(x),\left\{y_{0}, y_{1}\right\} \subset \Phi^{\gamma},\left\|y_{0}-y_{1}\right\|=\tau\right) .
\end{aligned}
$$

Note that the probability (28) is a function of $n, t_{0}, \tau$ and $\lambda^{\gamma}$; so it is convenient to use the function $\tilde{u}$ such that

$$
u(n, x, \tau, \lambda, \gamma)=\tilde{u}\left(n, 1-G_{\gamma}(x), \tau, \lambda^{\gamma}\right) .
$$

It is shown in Appendix VII that this function is given by (29) and it is shown at the bottom of this page. Unlike $h(\tau, \lambda)$ in (8) which is the function of only $\tau$ and $\lambda, \tilde{u}\left(n, t_{0}, \tau, \lambda\right)$ is a function of $n$ and $t_{0}$ (or $x$ ) as well, which means it can captures the impact of the number of neighboring nodes and the instantaneous channel. The impact of number of neighbors is shown in Fig. 2a and the impact of the channel (or timer value $\left.t_{0}\right)$ is shown in Fig. $2 \mathrm{~b}$.

Fig.2a exhibits plots for $\tilde{u}\left(n, t_{0}, \tau, \lambda\right)$ for $\lambda=1, v=0.5$, $t_{0}=0.5$ and for $n=0, \ldots, 20$. Observe how $\tilde{u}$ changes as the distance $\tau$ between $y_{0}$ and $y_{1}$ changes. As $\tau$ gets larger, $y_{1}$ behaves like a typical node in space which is not affected by the existence of $y_{0}$. The latter case is verified by the fact that all curves $\tilde{u}$ converge to the value $\frac{1-e^{-\bar{N}_{0}}}{\bar{N}_{0}}$ as $\tau \rightarrow \infty$, which is indeed the transmission probability of a typical CSMA node. As $\tau$ gets small, there is a strong correlation between $y_{1}$ and $y_{0}$ which are likely to be neighbors. The behavior of $\tilde{u}$ in this case depends on the value of $n$. In particular, if $n=0, \tilde{u}$ increases as $\tau \rightarrow 0$; since $y_{1}$ will see no contenders as is the case for $y_{0}$, while if $n>0$, as $\tau \rightarrow 0, y_{1}$ will see one or more contenders as seen by $y_{0}$, and it will be more likely that $y_{1}$ is a neighbor of $y_{0}$. If $y_{1}$ is a neighbor of $y_{0}$, then due to the condition $\left\{E_{0}=1\right\}, y_{1}$ must have a timer value larger than $t_{0}$, so the conditional transmission probability $\tilde{u}$ approaches 0 .

$$
\begin{aligned}
& \tilde{u}\left(n, t_{0}, \tau, \lambda\right)=\frac{\bar{N}_{0} G\left(\nu \tau^{\alpha}\right)}{n+\left(\bar{N}_{0}-n\right) G\left(\nu \tau^{\alpha}\right)}\left\{\frac{\left(1-e^{-t_{0} \bar{N}_{0}\left(1-p_{s}\right)}\right)}{\bar{N}_{0}\left(1-p_{s}\right)}\right. \\
& \left.+\left(1-t_{0}\right) e^{-\bar{N}_{0}\left(1-p_{s}\right)} \sum_{k=0}^{n} \frac{k !}{\eta^{k+1}}\left(1-e^{-\eta} \sum_{j=0}^{k} \frac{\eta^{j}}{j !}\right)\left(\begin{array}{l}
n \\
k
\end{array}\right) p_{s}^{k}\left(1-p_{s}\right)^{n-k}\right\},
\end{aligned}
$$

where $p_{s}=p_{s}(\tau)=2-\frac{b(\tau, \lambda)}{\bar{N}_{0}}$, and $\eta=\bar{N}_{0}\left(1-p_{s}\right)\left(t_{0}-1\right)$. 
As $n$ increases, $y_{1}$ is more likely to be preempted by $y_{0}$ and its neighbors, thus $\tilde{u}$ decreases.

Fig. $2 \mathrm{~b}$ shows the impact of $y_{0}$ 's timer value, $t_{0}$, on $\tilde{u}$ for $v=0.5$ and $n=5$. Note that the condition $\left\{E_{0}=1\right\}$ implies that $n$ neighbors of $y_{0}$ have timer values between $t_{0}$ and 1 . Thus, if $t_{0}$ gets large, $y_{1}$ will transmit with high probability since the neighbors of $y_{0}$ will have timer values larger than $t_{0}$, which can be easily preempted by $y_{1}$ 's timer. While if $t_{0}$ gets small, $y_{1}$ is more likely to be preempted by $y_{0}$ 's neighbors, so $\tilde{u}$ decreases in this case.

In summary, (26) can be approximated by

$$
\mathbb{E}^{0 !}\left[\mathbb{P}^{0 !}\left(F_{0, \gamma}^{\max }\left(N_{0}^{\gamma}\right)>\operatorname{tr}^{\alpha} I_{\Phi_{u}^{\gamma}} \mid N_{0}^{\gamma}, F_{0, \gamma}^{\max }\left(N_{0}^{\gamma}\right)\right)\right] .
$$

Let $\xi_{u}^{n, x}$ be the conditional pdf of $I_{\Phi_{u}^{\gamma 0 !}}$ given $N_{0}^{\gamma}=n$ and $F_{0}^{\max }\left(N_{0}^{\gamma}\right)=x$, so, (30) can be rewritten as

$$
\mathbb{E}^{0 !}\left[\int_{-\infty}^{\infty} \xi_{u}^{N_{0}^{\gamma}, F_{0, \gamma}^{\max }\left(N_{0}^{\gamma}\right)}(y) \mathbf{1}\left\{0 \leq y \leq \frac{F_{0, \gamma}^{\max }\left(N_{0}^{\gamma}\right)}{t r^{\alpha}}\right\} d y\right],
$$

where $\mathbf{1}\left\{0 \leq y \leq \frac{F_{0, \gamma}^{\max }\left(N_{0}^{\gamma}\right)}{t r^{\alpha}}\right\}$ and $\xi_{u}^{n, x}$ are both square integrable; see [4]. Applying the Plancherel-Parseval Theorem to evaluate the last equation, and performing a change of variables gives

$$
\begin{aligned}
p_{\text {suc }}^{q t}(\lambda, \gamma, v, t) \approx \mathbb{E}^{0 !}[ & \int_{-\infty}^{\infty} \mathcal{L}_{I_{I_{0}^{\gamma}, F_{0, \gamma}^{\max }\left(N_{0}^{\gamma}\right)}\left(2 i \pi r^{\alpha} t s\right)} \\
& \left.\times \frac{\exp \left\{2 i \pi s F_{0, \gamma}^{\max }\left(N_{0}^{\gamma}\right)\right\}-1}{2 i \pi s} d s\right] .
\end{aligned}
$$

Note that the expectation in (32) is with respect to $N_{0}^{\gamma}$ and $F_{0, \gamma}^{\max }\left(N_{0}^{\gamma}\right)$, and $I_{\Phi_{u}^{\gamma 0 !}}^{n, x}$ is a random variable with cdf $\mathbb{P}^{0 !}\left(I_{\Phi_{u}^{\gamma}}<\right.$ $\left.z \mid N_{0}^{\gamma}=n, F_{0}^{\max }\left(N_{0}^{\gamma}\right)=x\right)$. We have

$$
\begin{aligned}
& \mathcal{L}_{I^{n, x}, x !}(s) \\
& =\exp \left\{-\lambda^{\gamma} \int_{0}^{\infty} \int_{0}^{2 \pi} \frac{\tilde{u}\left(n, 1-G_{\gamma}(x), \tau, \lambda^{\gamma}\right) \tau d \theta d \tau}{1+\mu f(\tau, r, \theta) / s}\right\} .
\end{aligned}
$$

Replacing (33) into (32) gives the numerically computable approximation of $p_{s u c}^{q t}$.

\section{Spatial Reuse}

In this section, we compare the spatial reuse achieved by O-CSMA versus that of QT-CSMA. To better understand the results and the behavior of the protocols as a function of $\lambda, \gamma$, and $\nu$, we first study how transmission probability and success probability change as functions of the parameters, and then we compare the performance of O-CSMA and QT-CSMA. A brief performance comparison between O-ALOHA and O-CSMA follows.

\section{A. System Behavior and Parameter Sensitivity}

1) Density of Active Transmitters $\lambda p_{t x}$ : In Fig. 3, we show the density of active transmitters $\lambda p_{t x}$ as a function $\lambda$. As $\lambda$ increases, a higher number of active transmitters is achieved, which saturates to a value we will call the asymptotic density of active transmitters.

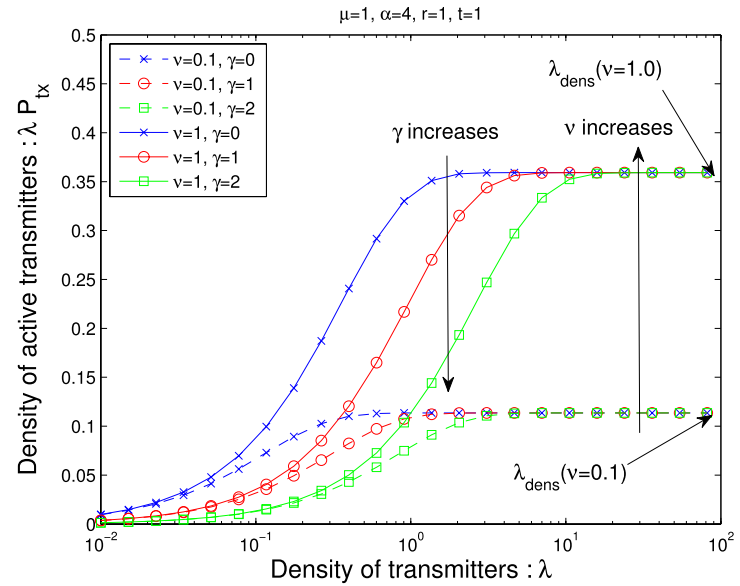

Fig. 3. The density of active transmitters for O/QT-CSMA increases and saturates as $\lambda$ increases due to the carrier sense in CSMA protocol. Increasing the qualification threshold $\gamma$ reduces the density of qualified transmitters without affecting the asymptotic density of active transmitters $\lambda_{\text {dens }}(v)$; so the effect is a shift of the curves to the right hand side. Increasing carrier sense threshold $v$ increases $\lambda_{\text {dens }}(v)$ since it makes the mean size of a typical transmitter's neighborhood smaller.

Definition 1 (Asymptotic Density of Active Transmitters): For a given carrier sense threshold $v$, the asymptotic density of active transmitters $\lambda_{\text {dens }}(v)$ is defined as

$$
\lambda_{\text {dens }}(\nu) \equiv \lim _{\lambda \rightarrow \infty} \lambda p_{t x}^{o p}(\lambda, \gamma, \nu)=\lim _{\lambda \rightarrow \infty} \lambda p_{t x}^{q t}(\lambda, \gamma, \nu) .
$$

Note that $\lambda_{\text {dens }}(v)$ is not the function of $\gamma$, since numerator $\exp \left\{-p_{\gamma} \bar{N}_{0}\right\}$ in $p_{t x}^{o p / q t}(\lambda, \gamma, \nu)$ vanishes as $\lambda \rightarrow \infty$; see (14) and (24). It is easy to show that $\lambda_{\text {dens }}(v)=1 / \hat{N}_{0}$, where $\hat{N}_{0}=\bar{N}_{0}^{\gamma} / \lambda^{\gamma}=\mathbb{E}\left[\int_{\mathbb{R}^{2}} \mathbf{1}\left\{F^{\prime}>v\|x\|^{\alpha}\right\} d x\right]$ is the mean neighborhood area of a typical transmitter. Note that since each active transmitter "occupies" an area of average size $\hat{N}_{0}$, intuitively, we can have at most $\frac{1}{\hat{N}_{0}}$ active transmitters per unit space in the asymptotically dense network (a network with $\lambda \rightarrow \infty$ ). Note that both O-CSMA and QT-CSMA have the same asymptotic density of transmitters $\lambda_{\text {dens }}(v)$ due to the transmitter selection process of the CSMA protocol.

As $\gamma$ increases, the density of qualified transmitters, $\lambda p_{\gamma}$, decreases, which accordingly decreases $\lambda p_{t x}$, but the limiting value $\lambda_{\text {dens }}(v)$ is not affected. As $v$ increases, the mean neighborhood area $\hat{N}_{0}$ gets smaller, which allows a higher density of active transmitters, and accordingly $\lambda_{\text {dens }}(v)$ increases as a function of $v$.

2) Success Probability of O-CSMA: Fig. 4a shows the success probability $p_{s u c}^{o p}(\lambda, \gamma, \nu, t)$ as a function of $\lambda$ for various $\gamma$ and $\nu$ values. The general behavior of $p_{s u c}^{o p}(\lambda, \gamma, v, t)$ is as follows. As $\gamma$ increases, the signal quality at receivers improves and at the same time the density of active transmitters goes down, which results in reduced interference at the receiver. Thus, increasing $\gamma$ increases SINR at receivers, and thus increases the success probability. If $v$ increases, the mean neighborhood area goes down, resulting in a higher number of active transmitters, which accordingly generate a stronger aggregate interference. Thus both the received SINR and success probability are decreased. As $\lambda$ increases the success probability $p_{s u c}^{o p}(\lambda, \gamma, v, t)$ converges to a value strictly less 


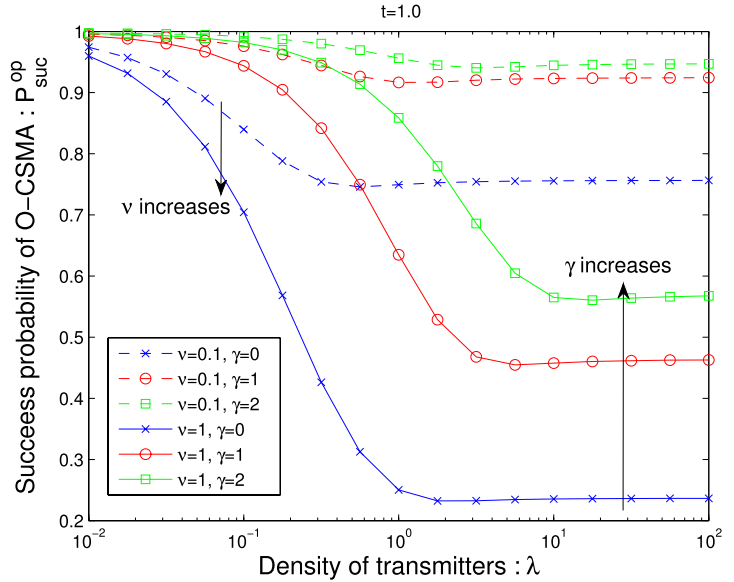

(a)

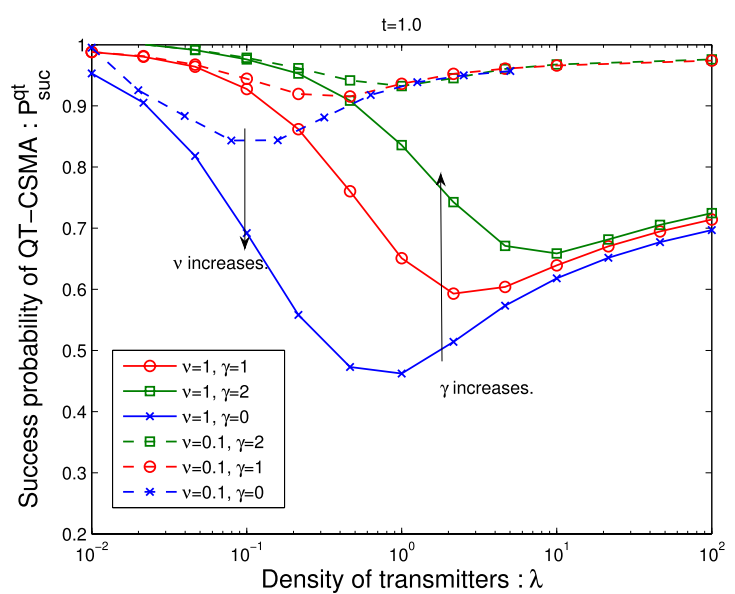

(b)

Fig. 4. The success probability versus the density of transmitters for various $v$ and $\gamma$. (a) The success probability of O-CSMA decreases as $\lambda$ increases, but converges to a value between 0 and 1 since interference

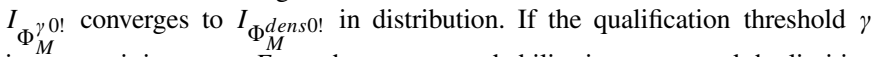
increases, it increases $F_{\gamma}$ so the success probability increases, and the limiting value $\lim _{\lambda \rightarrow \infty} p_{\text {suc }}^{o p}(\lambda, \gamma, v, t)$ also increases. However, if the carrier sense threshold $v$ increases, it increases the density of active transmitters, which accordingly increases interference, which deteriorates the success probability. (b) As $\lambda$ increases, the success probability of QT-CSMA decreases at first, but bounces and converges to 1 due to the increasing gain from opportunistic node selection. As the qualification threshold $\gamma$ increases, the success probability increases due to the improved channel quality. While, if carrier sense range $v$ increases, the success probability decreases due to increased aggregate interference power.

than 1 , i.e.,

$$
\lim _{\lambda \rightarrow \infty} p_{s u c}^{o p}(\lambda, \gamma, \nu, t)=\lim _{\lambda \rightarrow \infty} \mathbb{P}^{0 !}\left(F_{\gamma}>\operatorname{tr}^{\alpha} I_{\Phi_{M}^{\gamma}}\right)<1
$$

This is because $F_{\gamma}$ is exponentially distributed with an infinite support and $I_{\Phi_{M}^{\gamma 0 !}}$ converges in distribution to a random variable $I_{\Phi_{M}^{\text {dens } 0 !}} \equiv \sum_{i: X_{i} \in \Phi_{M}^{\text {dens } 0 !}} F_{i 0}\left\|X_{i}\right\|^{-\alpha}$, where $\Phi_{M}^{\text {dens } 0}$ is a Matérn CSMA point process with a density $\lambda_{\text {dens }}(v)$ given an active transmitter at the origin. The convergence of $I_{\Phi_{M}^{\gamma} \text { ! }}$ is formally shown in [16]. Since both random variables have infinite support in $\mathbb{R}_{+}, \mathbb{P}^{0 !}\left(F_{\gamma}>t r^{\alpha} I_{\Phi_{M}^{\gamma}}\right)$ converges to a positive value between 0 and 1 . It is not easy to find the limit since this would require characterizing $I_{\Phi_{M}^{\text {dens }} \text { ! . }}$

3) Success Probability of QT-CSMA: Fig. 4b shows the success probability $p_{s u c}^{q t}(\lambda, \gamma, \nu, t)$ as a function of $\lambda$ for various $\gamma$ and $v$ values. The general behavior of $p_{s u c}^{q t}(\lambda, \gamma, v, t)$ is as follows. As $\gamma$ increases, the interference seen at the receiver decreases due to the reduced density of active transmitters. However it is not clear how the received signal strength would change. Indeed, increasing $\gamma$ should shift $F_{\gamma}$ to the right hand side (improving the channel quality) but, at the same time, it decreases the size of neighborhood, thus reducing the opportunistic node selection gain. Fig. $4 \mathrm{~b}$ suggests that the positive effect is larger than the negative effect, and, as $v$ increases, $p_{s u c}^{q t}(\lambda, \gamma, v, t)$ decreases due to the increased interference. One thing to note is that if the density $\lambda$ becomes large enough, then the success probability increases and eventually converges to 1 due to the gain from opportunistic node selection with the best channel condition.

Precisely, if $\lambda \rightarrow \infty$ while $\nu, \gamma<\infty$ are kept fixed, we have

$$
\lim _{\lambda \rightarrow \infty} p_{s u c}^{q t}(\lambda, \gamma, v, t)=\lim _{\lambda \rightarrow \infty} \mathbb{P}^{0 !}\left(F_{\gamma}^{\max }\left(N_{0}^{\gamma}\right)>t r^{\alpha} I_{\Phi_{M}^{\gamma}}\right)=1 .
$$

This result can be intuitively understood as follows. As $\lambda$ increases, $N_{0}^{\gamma} \quad$ and $F_{\gamma}^{\max }\left(N_{0}^{\gamma}\right)$ increase (meaning $\lim _{\lambda \rightarrow \infty} \mathbb{P}\left(N_{0}^{\gamma}>x\right)=1$ and $\lim _{\lambda \rightarrow \infty} \mathbb{P}\left(F_{\gamma}^{\max }\left(N_{0}^{\gamma}\right)>x\right)=1$ for all fixed $x>0$ ), and $I_{\Phi_{M}^{\gamma 0} \text { ! converges in distribution }}$ to a random variable $I_{\Phi_{M}^{\text {dens } 0} \text {; }}$ see [16]. The success probability of O-CSMA and QT-CSMA are compared in the following proposition.

Proposition 4: Under the same parameter set $t, \gamma, v$, and $\lambda$, the success probability of QT-CSMA is never less than O-CSMA, i.e., $p_{\text {suc }}^{q t}(\lambda, \gamma, v, t) \geq p_{\text {suc }}^{o p}(\lambda, \gamma, v, t)$.

This directly follows from a stochastic ordering relation : $F_{\gamma}^{\max } \geq{ }^{s t} F_{\gamma}$; see (3).

Remark 1: Note that this implies that the density of successful transmissions of QT-CSMA is always higher than that of O-CSMA, i.e., $d_{s u c}^{q t}(\lambda, \gamma, v, t) \geq d_{s u c}^{o p}(\lambda, \gamma, v, t)$ for a given parameter set $t, \gamma, \nu$ and $\lambda$.

Remark 2: The above observations suggest that the effects of adjusting $\gamma$ and $v$ are similar in that both control the amount of interference in the network versus the opportunistic node selection gain which are achieved. However, this does not imply that O-CSMA can optimize its performance by optimizing only one of them while fixing the other, but interestingly this seems to work for QT-CSMA. In the following sections, we will further explore the possibility of reducing the number of parameters for QT-CSMA.

\section{B. Performance Comparison of O-CSMA and $Q T_{0}-C S M A$}

We evaluate the performance of a network under various system parameters and suggest a reasonable choice of $v$ which makes $\mathrm{QT}_{0}$-CSMA robust to changes in the environment ${ }^{9}$. Note that it is no surprise to find that QT-CSMA always does better than O-CSMA under the same parameter set as shown in Proposition 4. Thus, we focus instead on the comparison between $\mathrm{QT}_{0}$-CSMA (QT-CSMA with $\gamma=0$ ) and O-CSMA.

\footnotetext{
${ }^{9}$ In this work we assume that $t$ and $r$ are fixed. $t$ is determined from a given transmission rate requirement and $r$ is simply assumed to be fixed for mathematical simplicity.
} 


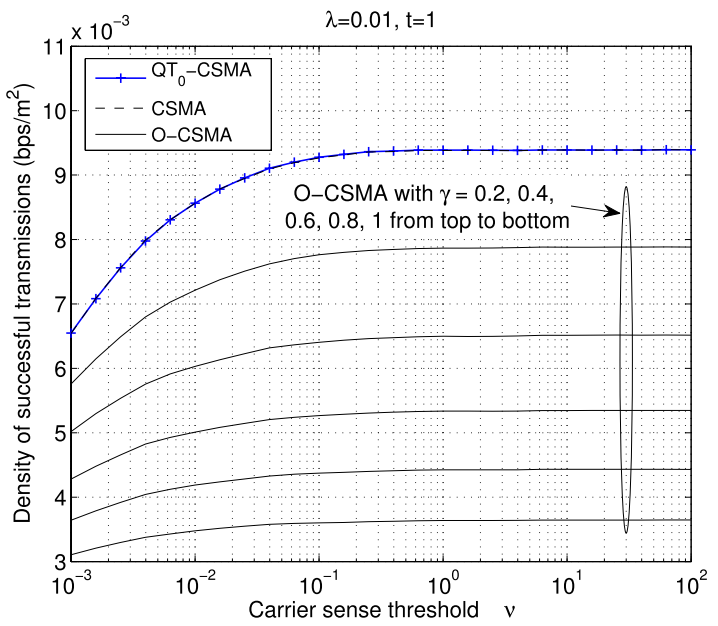

(a)

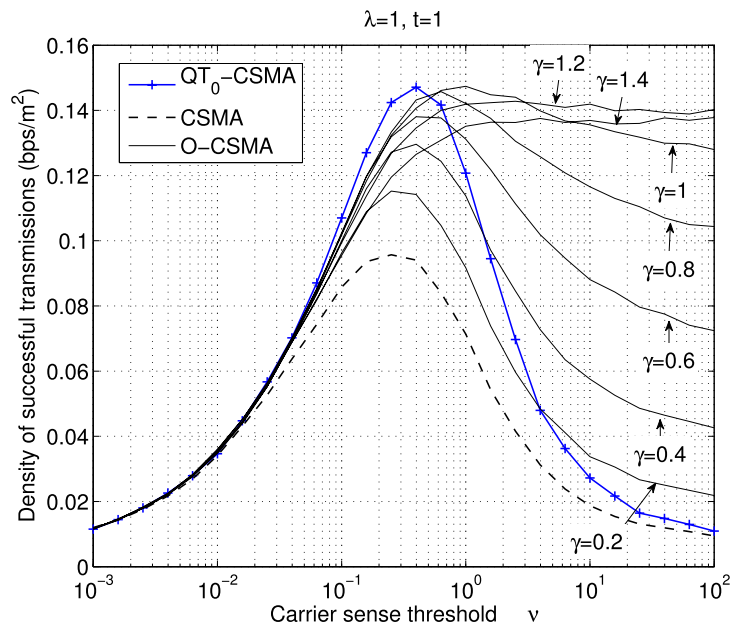

(c)

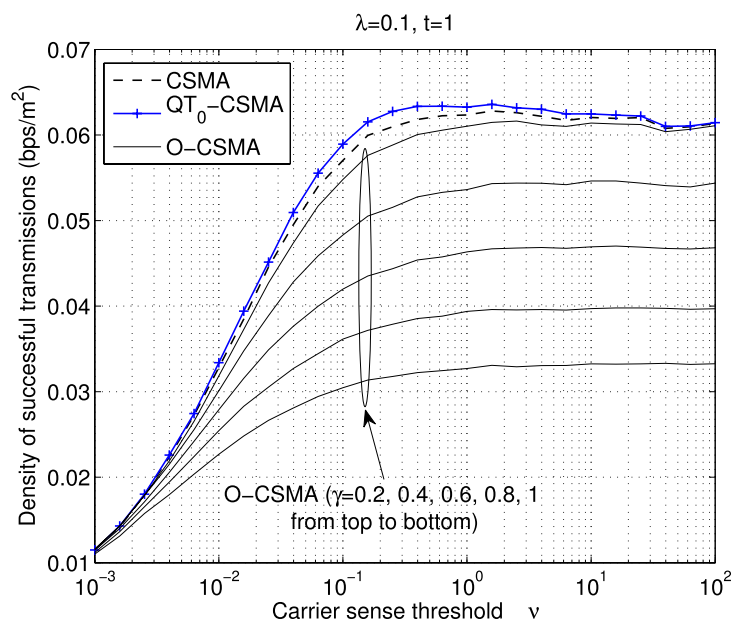

(b)

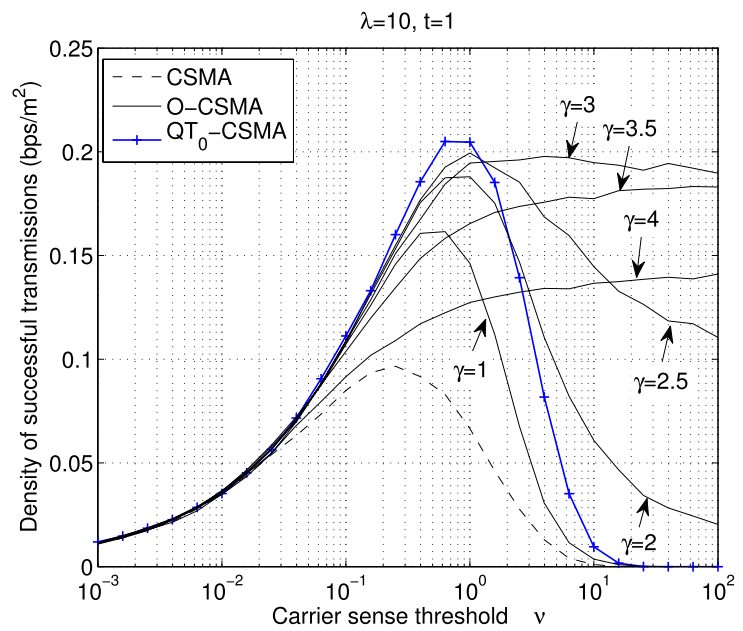

(d)

Fig. 5. The density of successful transmissions for QT-CSMA, QT 0 -CSMA, and O-CSMA versus $v$ were shown under various values of $\gamma$ for $\lambda=0.01$, $0.1,1$, and 10. $v=0.5$ is a good choice for both $\mathrm{QT}_{0}$-CSMA and O-CSMA under various $\gamma$. Only simulations results were used for choosing the suggested $v$ value.

1) Choosing Carrier Sense Threshold v: The carrier sense threshold $v$ controls the size of the virtual exclusion region around transmitters inside which no other transmitters will transmit. The size of the guard zone is directly related to the spatial reuse through the density of active nodes and generated network interference. Large $v$ (or small guard zone) increases the density of active transmitters, but at the same time it could introduce strong network interference. One the other hand, small $v$ (or large guard zone) induces the low density of concurrent active transmitters, which results in weak network interference and accordingly high transmission success probability. Thus, selecting appropriate $v$ is very important for network performance. The difficult part is that this is an optimization problem over two variables $v$ and $\gamma$. However, we show that there exist a good choice of $v$ which is mostly insensitive to the change of $\gamma$.

In the sequel we show that $v=0.5$ is a reasonable choice for both $\mathrm{QT}_{0}$-CSMA and O-CSMA. In Fig. 5, the density of successful transmissions for $\mathrm{QT}_{0}$-CSMA and
O-CSMA/QT-CSMA are shown for $\lambda=0.01,0.1,1$, and 10 . The optimal $v$ maximizing the spatial reuse of $\mathrm{QT}_{0}$-CSMA depends on $\lambda$. However, $v=0.5$ is a near optimal choice for all $\lambda$. The optimal $v$ for O-CSMA also depends on $\lambda$. When $\lambda$ $=0.01$ or 0.1 , any $v$ greater than 0.1 is a reasonable choice. While, when $\lambda=1$ or 10 , the optimal $v$ depends on the choice of $\gamma$; optimal $v$ for $\lambda=1$ increases from 0.2 (when $\gamma=0.2$ ) to 0.9 (when $\gamma=1$ ), and optimal $v$ for $\lambda=10$ increases from 0.4 (when $\gamma=0.5$ ) to 2 (when $\gamma=2.5$ ). Even with this dependency, $v=0.5$ for O-CSMA is a good choice for the $\gamma$ values. Based on these observations, we argue that $v=0.5$ is a choice which not only results in high spatial reuse but also makes protocols robust to a wide range of $\gamma$ and $\lambda$.

2) Choosing the Qualification Threshold $\gamma$ : Once the carrier sense threshold $v$ is chosen, the qualification threshold $\gamma$ can be chosen for the given $v$. In Fig. 6, the impact of the qualification threshold $\gamma$ on the spatial reuse is shown for various $\lambda$ values. In a sparse network $(\lambda=0.1)$, it is better not to have the qualification process since it reduces the 


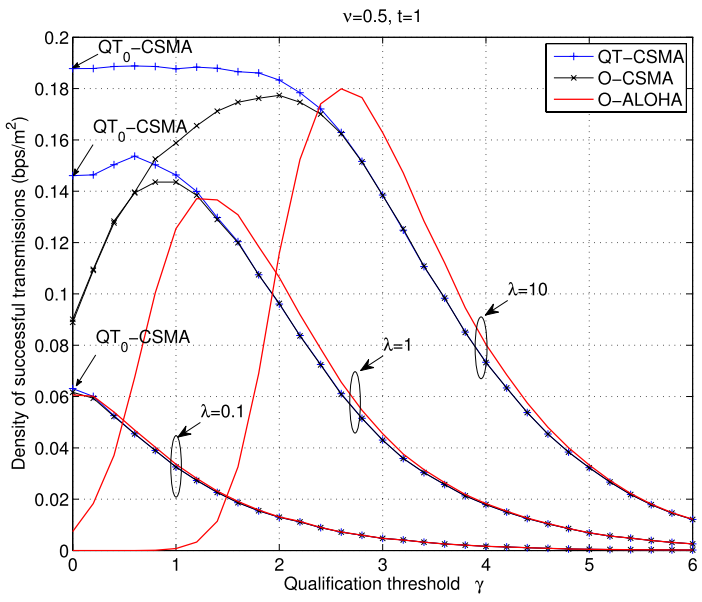

Fig. 6. The impact of qualification threshold $\gamma$ on the density of successful transmissions is plotted for various $\lambda$. In sparse network $(\lambda=0.1)$ setting $\gamma=0$ maximizes spatial reuse since node density is low. In dense networks, there exist an optimal $\gamma$ for O-CSMA (black solid lines) which is the function of network density. Note that QT-CSMA (blue lines with cross marker) with $\gamma=0$ corresponds to $\mathrm{QT}_{0}$-CSMA. Note that the spatial reuse of $\mathrm{QT}_{0}-\mathrm{CSMA}$ is quite high even without qualification process. In this figure, only simulations results were shown.

density of active transmitters. The loss in the number of active transmitters due to increased $\gamma$ is larger than the gain from the increased success probability. Note that it also applies to sparse networks with $\lambda<0.1$.

For an intermediate density network $(\lambda=1)$, it is required to optimize $\gamma$ for O-CSMA (and for O-ALOHA as well) to improve spatial reuse. However, unlike O-CSMA, optimizing $\gamma$ for QT-CSMA does not give much improvement. Setting $\gamma=0$ is a simple but effective solution. Note that it also applies to intermediate networks with $\lambda \approx 1$.

In a dense network $(\lambda=10)$, O-CSMA requires the optimization of $\gamma$, while QT-CSMA does not; simply setting $\gamma=0$ is an optimal choice. Note that it also applies to dense networks with $\lambda>10$. Through above cases, we observe that $\gamma$ needs to be optimized for O-CSMA (and O-ALOHA as well as shown in Fig. 6) to achieve high spatial reuse. However, the optimal $\gamma$ depends on $\lambda$. Considering the difficulty of estimating $\lambda$ in practice and likelihood the density is non-homogeneous, optimizing $\gamma$ for O-CSMA is not a practical approach. In this sense, $\mathrm{QT}_{0}-\mathrm{CSMA}$ is an attractive engineering choice since it does not require the optimization of $\gamma$ while providing reasonably high performance which is as high as the maximum performance of QT-CSMA. Due to the absence of qualification process, $\mathrm{QT}_{0}$-CSMA is easy to configure and robust to changes in $\lambda$.

3) Robustness of $Q T_{0}$-CSMA: Fig. 7 exhibits the above data from a different perspective: the density of successful transmissions for $\mathrm{QT}_{0}$-CSMA and O-CSMA versus $\lambda$. In Fig. 7 we plot both analysis and simulation results to show how well they match. For comparison, ALOHA, CSMA and O-ALOHA are also plotted using our model. The figure confirms the behavior of ALOHA for increasing node density. Unlike ALOHA, the density of successful transmissions of CSMA does not converge to 0 as $\lambda$ increases due to carrier sensing and controlled network interference. As mentioned

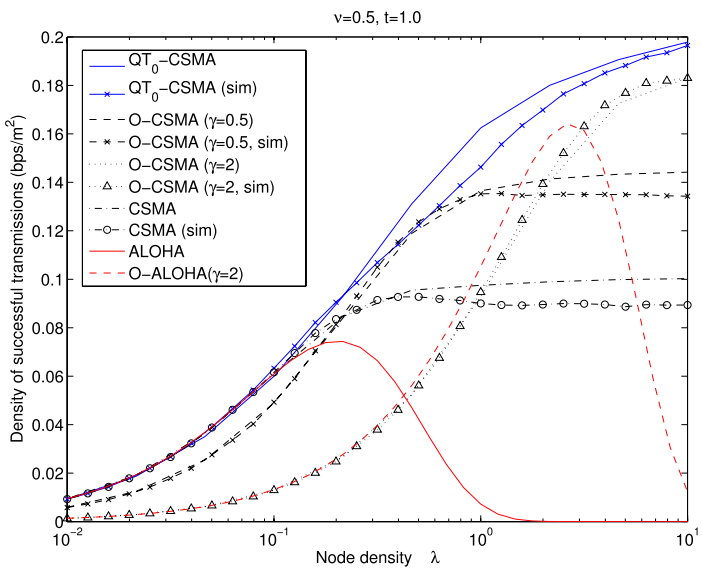

Fig. 7. The density of successful transmissions versus $\lambda$ was shown for $v=0.5$. We plotted both simulation and analytical results for comparison. In case of (O-)ALOHA, we plotted only analytical results since simulation results were identical.

earlier, to take advantage of channel variations, ALOHA can qualify users based on channel threshold, which we call O-ALOHA.

O-CSMA with a similar threshold mechanism works as follows. As $\lambda$ gets larger, $d_{s u c}^{o p}(\gamma, \lambda)$ increases as the result of the increasing density of active transmitters; however it converges to fixed values since both the density of active transmitters and success probability converge. If $\lambda$ gets large, $d_{\text {suc }}^{o p}$ increases and converges to a value less than $\lambda_{\text {dens }}$.

$\mathrm{QT}_{0}$-CSMA performs better than O-CSMA for all $\lambda$ values. This proves that the robustness of $\mathrm{QT}_{0}-\mathrm{CSMA}$; quantile-based scheduling without qualification can fully take advantage of opportunistic node selection gains in the wide range of $\lambda$ provided that $v$ is properly chosen.

\section{SPATIAL FAIRNESS}

\section{A. Unfairness in CSMA Networks}

In this section, we compare the degree of "spatial fairness" achieved by O/QT-CSMA protocols. It has been reported that non-slotted CSMA networks are unfair [27], [28]. The two main reasons are the irregularity in the network topology and protocol behaviors that lead to starvation for some nodes. There have been efforts towards improving fairness by tuning protocols, for example, adjusting carrier sense range [29] or using node specific access intensity [28], [30], [31].

In slotted systems, unfairness is reduced since all nodes' contention windows are reset every slot, which prevents starvation. However, unfairness due to irregularities in network topologies remains. We will show in this section that our opportunistic scheduling schemes can improve fairness.

\section{B. Spatial Fairness}

We define two spatial fairness indices which capture a fairness of the long-term (time-averaged) performance across nodes in space. The first captures the heterogeneity in performance due to nodes' locations. Recall that the performance of node, say $X_{i}$, is affected by the remaining nodes and 
their locations, i.e. $\Phi \backslash\left\{X_{i}\right\}$ and channel gains $\mathbf{F}_{i}$ and $\mathbf{F}_{i}^{\prime}$. Let $f_{i}\left(\Phi, \mathbf{F}_{i}, \mathbf{F}_{i}^{\prime}\right)$ be a bounded function associated with $X_{i} \in \Phi$ denoting its performance. Then, $\mathbb{E}\left[f_{i}\left(\Phi, \mathbf{F}_{i}, \mathbf{F}_{i}^{\prime}\right) \mid \Phi=\phi\right]$ denotes the time-average (or equivalently, the average w.r.t. $\mathbf{F}_{i}$ and $\mathbf{F}_{i}^{\prime}$ ) of $X_{i}$ 's performance given $\Phi=\phi$. To evaluate the fairness of $\mathbb{E}\left[f_{i}\left(\Phi, \mathbf{F}_{i}, \mathbf{F}_{i}^{\prime}\right) \mid \Phi=\phi\right]$ across nodes $X_{i} \in \Phi=\phi$ in space we introduce Jain's fairness index ${ }^{10}$, where

$$
\mathrm{FI}=\lim _{l \rightarrow \infty} \frac{\left(\sum_{i: X_{i} \in \phi \cap B_{l}} \mathbb{E}\left[f_{i}\left(\Phi, \mathbf{F}_{i}, \mathbf{F}_{i}^{\prime}\right) \mid \Phi=\phi\right]\right)^{2}}{\left|\phi \cap B_{l}\right| \sum_{i: X_{i} \in \phi \cap B_{l}}\left(\mathbb{E}\left[f_{i}\left(\Phi, \mathbf{F}_{i}, \mathbf{F}_{i}^{\prime}\right) \mid \Phi=\phi\right]\right)^{2}} .
$$

Given the spatial ergodicity of homogeneous PPPs; see [25], and simple algebra, it is easy to see that (36) becomes

$$
\mathrm{FI}=\frac{\left(\mathbb{E}^{0}\left[\mathbb{E}\left[f_{0}\left(\Phi, \mathbf{F}_{0}, \mathbf{F}_{0}^{\prime}\right) \mid \Phi\right]\right]\right)^{2}}{\mathbb{E}^{0}\left[\left(\mathbb{E}\left[f_{0}\left(\Phi, \mathbf{F}_{0}, \mathbf{F}_{0}^{\prime}\right) \mid \Phi\right]\right)^{2}\right]},
$$

where $\mathbf{F}_{0}$ and $\mathbf{F}_{0}^{\prime}$ denote the channel fading of a typical node at the origin and accordingly $\mathbb{E}\left[f_{0}\left(\Phi, \mathbf{F}_{0}, \mathbf{F}_{0}^{\prime}\right) \mid \Phi\right]$ denotes the performance seen by the node $X_{0}$.

The second fairness index captures the heterogeneity in performance across nodes seeing different neighborhood size. We, let $\tilde{f}_{i}\left(N_{i}, \mathbf{F}_{i}, \mathbf{F}_{i}^{\prime}\right)$ be a finite performance metric associated with $X_{i}$, where $N_{i}$ is the number of neighbors of $X_{i}$. Then, $\mathbb{E}\left[\tilde{f}_{i}\left(N_{i}, \mathbf{F}_{i}, \mathbf{F}_{i}^{\prime}\right) \mid N_{i}=n\right]$ denotes the time-averaged (or $\mathbf{F}_{i}$ and $\mathbf{F}_{i}{ }^{\prime}$-averaged) value associated with $X_{i}$ given $X_{i}$ has a neighborhood of size $N_{i}=n$. The corresponding Jain's fairness index is given by

$$
\tilde{F} I=\frac{\left(\mathbb{E}^{0}\left[\mathbb{E}\left[\tilde{f}_{0}\left(N_{0}, \mathbf{F}_{0}, \mathbf{F}_{0}^{\prime}\right) \mid N_{0}\right]\right]\right)^{2}}{\mathbb{E}^{0}\left[\left(\mathbb{E}\left[\tilde{f}_{0}\left(N_{0}, \mathbf{F}_{0}, \mathbf{F}_{0}^{\prime}\right) \mid N_{0}\right]\right)^{2}\right]} .
$$

Unlike (37), (38) does not capture a performance variability across nodes with the same number of contenders. However, (38) is a useful metric which is computable in many cases. Depending on the performance metric $f()$ of interest, we sometimes have FI $=\tilde{\text { FII }}$. In the sequel, we will focus on $\tilde{F I}$ as our measure of spatial fairness.

\section{Spatial Fairness for Conditional Access Probability}

We first evaluate spatial fairness for nodes' conditional access probability. We will show how nodes' random locations impact this metric. We need the following assumption.

Assumption 1 (Contention Neighborhood Based on Mean Channel Gain): We assume that $F_{i j}^{\prime}$ is deterministic with $F_{i j}^{\prime}=$ $\frac{1}{\mu}$, i.e, the contenders of node $X_{i}$ are the set of nodes located in the disc $b\left(X_{i},(v \mu)^{-\alpha}\right)$.

Under this assumption, the neighbors of a node are not affected by fading, so the size of a node's neighborhood stays fixed, e.g., might be based on the average channel gain.

\footnotetext{
${ }^{10}$ Jain's fairness index for a given positive allocation $x=\left(x_{i}: i=\right.$ $1, \ldots, n)$ is given as $\mathrm{FI}_{x}=\frac{\left(\sum_{i=1}^{n} x_{i}\right)^{2}}{n \sum_{i=1}^{n} x_{i}^{2}}$. Note that the maximum value of FI is 1 which is achieved when all $x_{i}$ s have the same value. If total resource $b=\sum_{i=1}^{n} x_{i}$ is allocated equally only to $k$ entities out of $n$, e.g, $x_{i}=\frac{b}{k}$ for $i=1, \ldots, k$ and $x_{i}=0$ for $i=k+1, \ldots, n$, then, we have $\mathrm{FI}_{x}=k / n$. See [32].
}

This might be a reasonable assumption in a system, where each node's contending neighbors are dynamically maintained based on the average fading gains to the node. Note that $F_{i j}$ is still a random variable, i.e., only the fading between transmitters has been changed. Let

$$
N_{s, i}^{\gamma}=N_{s, i}^{\gamma}(\Phi)=\left|\left\{X_{j} \in \Phi^{\gamma}: 1 /\left(\mu\left\|X_{i}-X_{j}\right\|^{\alpha}\right)>v, i \neq j\right\}\right|
$$

be a random variable denoting the size of $X_{i}$ 's neighborhood under the static fading Assumption $1^{11}$, it corresponds to the number of nodes inside a disk $b\left(X_{i},(v \mu)^{-\frac{1}{\alpha}}\right)$. This is a Poisson random variable with mean $\lambda \pi(v \mu)^{-\frac{2}{\alpha}}$. Recall that a node with $n$ contenders accesses the channel with probability $\frac{p_{\gamma}}{n+1}$. This corresponds to the fraction of time the node accesses the channel. We will call this quantity the conditional access probability of the node to differentiate it from the access probability (e.g., $p_{t x}^{o p}$ or $p_{t x}^{q t}$ ) which is interpreted as the fraction of nodes transmitting in space in a typical slot. Note that since the conditional access probability depends only on $N_{s, i}^{\gamma}$, so we have that $\mathbb{E}\left[f_{i}\left(\Phi, \mathbf{F}_{i}, \mathbf{F}_{i}^{\prime}\right) \mid \Phi\right]=$ $\mathbb{E}\left[\tilde{f}_{i}\left(N_{s, i}^{\gamma}, \mathbf{F}_{i}, \mathbf{F}_{i}^{\prime}\right) \mid N_{s, i}^{\gamma}\right]=\frac{p_{\gamma}}{N_{s, i}^{\gamma}+1}$. Thus we have following lemma regarding spatial fairness index on conditional access probability.

Lemma 1: If $\tilde{f}_{i}\left(N_{s, i}^{\gamma}, \mathbf{F}_{i}, \mathbf{F}_{i}^{\prime}\right)=\mathbf{1}\left\{F_{i i}>\gamma, E_{i}=1\right\}$, or equivalently $\mathbb{E}\left[\tilde{f}_{i}\left(N_{s, i}^{\gamma}, \mathbf{F}_{i}, \mathbf{F}_{i}^{\prime}\right) \mid N_{s, i}^{\gamma}\right]=\frac{p_{\gamma}}{N_{s, i}^{\gamma}+1}$ under the Assumption 1, the two spatial fairness indices are equal as follows:

$$
\begin{aligned}
\mathrm{FI}_{a c}=\tilde{F} I_{a c} & =\frac{\left(\mathbb{E}^{0}\left[\frac{p_{\gamma}}{N_{s, 0}^{\gamma}+1}\right]\right)^{2}}{\mathbb{E}^{0}\left[\left(\frac{p_{\gamma}}{N_{s, 0}^{\gamma}+1}\right)^{2}\right]} \\
= & \frac{e^{\bar{N}_{s, 0}^{\gamma}+e^{-\bar{N}_{s, 0}^{\gamma}}-2}}{\bar{N}_{s, 0}^{\gamma}\left(E i\left(\bar{N}_{s, 0}^{\gamma}\right)-\log \bar{N}_{s, 0}^{\gamma}-\eta\right)},
\end{aligned}
$$

where $\bar{N}_{s, 0}^{\gamma}=\mathbb{E}\left[N_{s, 0}^{\gamma}\right], E i(x)=-\int_{-x}^{\infty} t^{-1} e^{-t} d t$ is the exponential integral function, and $\eta=0.5772 \ldots$ is the EulerMascheroni constant. Note that these fairness indices are the function of $\bar{N}_{s, 0}^{\gamma}$.

Proof is given in Appendix VIII.

Fig.8a shows the fairness index for the conditional access probability under O/QT-CSMA versus $\bar{N}_{s, 0}^{\gamma}(v)$. If $\bar{N}_{s, 0}^{\gamma}(v)$ is small, almost every contending node is selected for transmission. Transmitters have conditional access probability close to $p_{\gamma}$, so that the fairness index is close to 1 . If $\bar{N}_{s, 0}^{\gamma}(v)$ is relatively small, as $\bar{N}_{s, 0}^{\gamma}$ (which is mean and the variability of the number of contenders) increases, the variability in the conditional access probability, across nodes increases resulting in a decrease in fairness. However, if $\bar{N}_{s, 0}^{\gamma}(v)$ is moderate, the fairness index eventually increases again since, in this regime, the variability of the conditional access probability $\frac{p_{\gamma}}{N_{s, 0}^{\gamma}+1}$ decreases and converges to 0 , which in turn increases fairness. Note that the fairness curve has a minimum of approaching $\approx 0.73$ when $\bar{N}_{s, 0}^{\gamma} \approx 3$.

\footnotetext{
${ }^{11}$ Note the difference between $N_{s, i}^{\gamma}$ and $N_{i}^{\gamma}$, where the latter is the number of neighbors without the static fading assumption.
} 


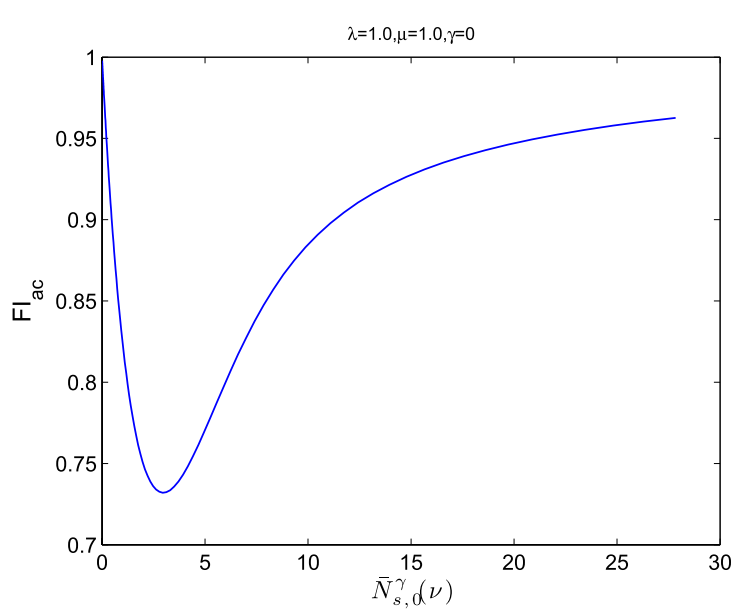

(a)

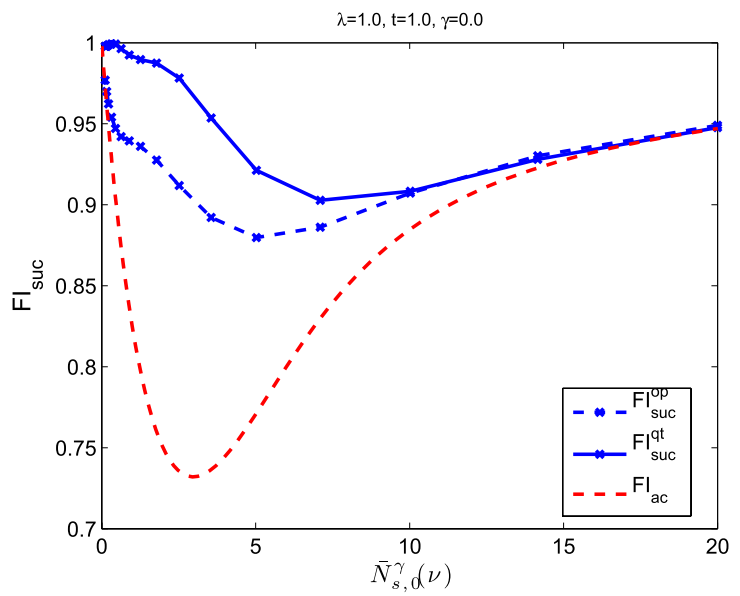

(b)

Fig. 8. Fairness index on conditional access probability and successful transmission versus the mean number of contenders $\bar{N}_{s, 0}^{\gamma}(v)$ under static fading vector assumption. (a) The fairness index on conditional access probability decreases as the mean number of contenders $\bar{N}_{s, 0}^{\gamma}$ decreases, but it rises soon as $\bar{N}_{s, 0}^{\gamma}$ increases. The fairness has minimum value $\sim 0.73$ when $\bar{N}_{s, 0}^{\gamma} \approx 3$. This applies to both O-CSMA and QT-CSMA. (b) Quantile scheduling increases fairness significantly because, under QT-CSMA, nodes with larger neighborhood size have a higher success probability, which compensates its low conditional access probability.

\section{Spatial Fairness of the Conditional Probability of Successful Transmissions}

In this section, we consider fairness for the conditional probability of successful transmissions. Specifically, we show that opportunistic CSMA schemes can, to a certain extent, remove topological unfairness. We first define spatial fairness for the conditional probability of successful transmissions.

For O-CSMA, we define $\frac{p_{\gamma}}{n+1} \bar{p}_{s u c}^{o p}(\gamma, n)$ as the conditional probability of successful transmissions of a typical transmitter with $n$ neighbors, where $\frac{p_{\gamma}}{n+1}$ is the conditional access probability and $\bar{p}_{s u c}^{o p}(\gamma, n)$ is the conditional success probability conditioned on the transmitter having $n$ contenders, which is given by

$$
\begin{aligned}
& \bar{p}_{s u c}^{o p}(\gamma, n)=\mathbb{P}^{0 !}\left(F_{\gamma}>t I_{\Phi_{M}^{\gamma}} r^{\alpha} \mid N_{s, 0}^{\gamma}=n\right), \\
& \approx \mathbb{E}_{F_{\gamma}}^{0 !}\left[\int_{-\infty}^{\infty} \mathcal{L}_{I_{\Phi_{u}^{\prime}}^{n, F_{\gamma}}}\left(2 i \pi r^{\alpha} t s\right) \frac{e^{2 i \pi s F_{\gamma}}-1}{2 i \pi s} d s\right],
\end{aligned}
$$

where $I_{\Phi_{u}^{\prime 0 !}}^{n, F_{\gamma}}$ is the interference seen by a typical receiver conditioned on that its associated transmitter has $n$ neighbors. Accordingly, the fairness index is given by

$$
\tilde{\mathrm{FI}}_{s u c}^{o p}=\frac{\left(\mathbb{E}^{0}\left[\frac{p_{\gamma}}{N_{s, 0}^{\gamma}+1} \bar{p}_{s u c}^{o p}\left(\gamma, N_{s, 0}^{\gamma}\right)\right]\right)^{2}}{\mathbb{E}^{0}\left[\left(\frac{p_{\gamma}}{N_{s, 0}^{\gamma}+1} \bar{p}_{s u c}^{o p}\left(\gamma, N_{s, 0}^{\gamma}\right)\right)^{2}\right]} .
$$

For QT-CSMA, we take a similar approach. We define $\frac{p_{\gamma}}{n+1} \bar{p}_{s u c}^{q t}(\gamma, n)$ as the conditional probability of successful transmission of a typical transmitter with $n$ neighbors, where $\frac{p_{\gamma}}{n+1}$ is the conditional access probability and $\bar{p}_{s u c}^{q t}(\gamma, n)$ is the conditional success probability of a typical receiver conditioned on that its associated transmitter has $n$ contenders, which is given by

$$
\begin{aligned}
\bar{p}_{s u c}^{q t}(\gamma, n) & =\mathbb{P}^{0 !}\left(F_{0, \gamma}^{\max }\left(N_{s, 0}^{\gamma}\right)>t I_{\Phi_{M}^{\gamma}} r^{\alpha} \mid N_{s, 0}^{\gamma}=n\right) . \\
& \approx \mathbb{E}^{0 !}\left[\int_{-\infty}^{\infty} \mathcal{L}_{n, F_{\Phi_{u}^{\prime}, \gamma}^{\max }(n)}\left(2 i \pi r^{\alpha} t s\right) \frac{e^{2 i \pi s F_{0, \gamma}^{\max }(n)}-1}{2 i \pi s} d s\right] .
\end{aligned}
$$

The fairness metric we use in this section corresponds to the second type (38) only, and the corresponding fairness index of successful transmission is given by

$$
\tilde{\mathrm{F}}_{s u c}^{q t}=\frac{\left(\mathbb{E}^{0}\left[\frac{p_{\gamma}}{N_{s, 0}^{\gamma}+1} \bar{p}_{s u c}^{q t}\left(\gamma, N_{s, 0}^{\gamma}\right)\right]\right)^{2}}{\mathbb{E}^{0}\left[\left(\frac{p_{\gamma}}{N_{s, 0}^{\gamma}+1} \bar{p}_{s u c}^{q t}\left(\gamma, N_{s, 0}^{\gamma}\right)\right)^{2}\right]} .
$$

Using (33) and $N_{s, 0}^{\gamma} \sim \operatorname{Poisson}\left(\bar{N}_{s, 0}^{\gamma}\right), \tilde{\mathrm{FI}}_{s u c}^{q t}$ can be numerically computed.

$\tilde{\mathrm{F}}_{s u c}^{o p}$ and $\tilde{\mathrm{FI}}_{\text {suc }}^{q t}$ are plotted in Fig.8b for $\gamma=0$. The figure shows that the spatial fairness on the conditional access probability of successful transmissions achieved by $\mathrm{QT}_{0}$-CSMA is improved versus that of O-CSMA. The gain is significant in the regime where $\bar{N}_{s, 0}^{\gamma}$ is less than or equal to roughly 10. In this regime, $\mathrm{QT}_{0}$-CSMA increases the success probability of receivers a lot. This reduces the performance differences among nodes caused by different conditional access probabilities(or topologies) since nodes with a large number of neighbors and low conditional access probability have a higher success probability. In other words, the higher success probability compensates the low conditional access probability, which decreases the variability in performance. In the regime where $\bar{N}_{s, 0}^{\gamma}$ is large (or $v$ is small), the density of concurrent transmitters becomes small, which generates weak interference. Thus, most nodes succeed in their transmissions with high probability irrespective of the number of neighbors, so in this regime there is no much gain from opportunism increasing the success probability. Thus, $\mathrm{QT}_{0}$-CSMA and O-CSMA have almost the same performance. As $\gamma$ increases, fairness decreases and eventually converges to the fairness curve of O-CSMA where $\gamma \rightarrow \infty$ since there is little difference between $\bar{p}_{\text {suc }}^{q t}$ and $\bar{p}_{\text {suc }}^{o p}$. 
So far, we have shown that opportunistic CSMA can improve fairness. However, with this result only, it is not clear how these protocols tradeoff the density of successful transmissions versus fairness. We consider this next.

\section{E. Tradeoff Between Spatial Fairness and Spatial Reuse}

In this section we consider the tradeoff between spatial reuse and spatial fairness which is due to the randomness of node locations, contention and protocols. To explore this, we introduce following notions.

- (FD-Fair) We call $(a, b)$ an achievable FD-pair if a fairness index $a$ and density of successful transmissions $b$ can be achieved for a given protocol parameter choice.

- (Dominance) For FD-pairs $(a, b)$ and $(c, d) \in \mathbb{R}_{+}^{2}$, we say that the $(a, b)$ dominates $(c, d)$ if $a \geq c$ and $b \geq d$. This relation is denoted by $(c, d) \preceq(a, b)$.

- (Dominated set) For a given FD-pair $(a, b)$, the set of FD-pairs dominated by $(a, b)$ is defined as follows.

$$
\Lambda(a, b)=\left\{(x, y) \in \mathbf{R}_{+}^{2} \text { s.t. }(x, y) \preceq(a, b)\right\}
$$

Note that $(a, b) \in \Lambda(a, b)$. In particular, we define the dominated set for O-CSMA, for a given $t$ and $\lambda$, by

$$
\Omega^{o p}(\lambda, t)=\bigcup_{\gamma \geq 0, \nu \geq 0} \Lambda\left(\tilde{F I}_{s u c}^{o p}(\lambda, \gamma, \nu, t), d_{s u c}^{o p}(\lambda, \gamma, \nu, t)\right) .
$$

The dominated set for QT-CSMA is similarly defined. The dominated set $\mathrm{QT}_{0}$-CSMA for a given $t$ and $\lambda$ is defined as

$$
\Omega_{0}^{q t}(\lambda, t)=\bigcup_{v \geq 0} \Lambda\left(\tilde{\mathrm{F}}_{\text {suc }}^{q t}(\lambda, 0, v, t), d_{s u c}^{q t}(\lambda, 0, v, t)\right)
$$

Three dominated sets for $\lambda=1$, decoding SIR target $t=1$ are exhibited in Fig. 9. The area surrounded by red dashed curve, the solid black curve, and the dotted blue curve, denotes the dominated set of $\mathrm{QT}_{0}$-CSMA, QT-CSMA, and O-CSMA respectively. In Fig. 9, we plotted several curves of pairs $\left(\tilde{\mathrm{FI}}_{s u c}(\lambda, \gamma, v, t), d_{s u c}\right)$ for O-CSMA to show how we computed the dominated set of O-CSMA. Each curve was drawn for various $v$ values from 0.02 to 612 for a given $\gamma$. We then computed the dominated set of the union of the curves. Note that we used spatial reuse results from simulation for accuracy, and spatial fairness from analytical computation since it is too hard to get reliable statistics for spatial fairness from simulation.

Note that the dominated set of QT-CSMA is larger than (dominates) that of O-CSMA. This gain comes from the joint improvement of spatial reuse and fairness performance. Also notable is that the dominated set of $\mathrm{QT}_{0}$-CSMA is quite large although it has one less parameter. This shows again the effectiveness of quantile-based approach in taking advantage of dynamic channel variations and multi-user diversity.

\section{CONCLUSION}

In this paper, we considered spatial reuse and fairness for wireless ad-hoc networks using two different channel-aware CSMA protocols. We used an analytical framework based

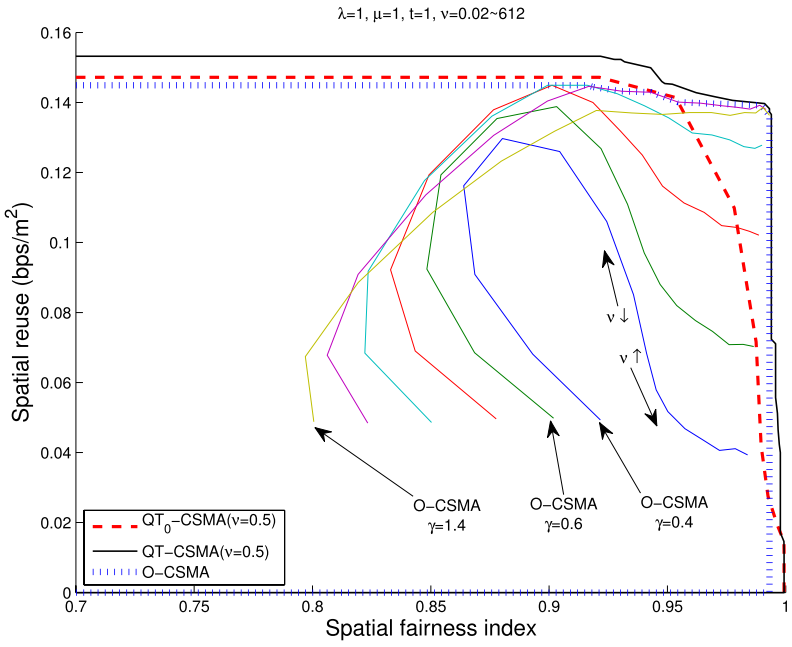

Fig. 9. Comparison of the dominated sets of O-CSMA, QT-CSMA and $\mathrm{QT}_{0}$-CSMA.

on stochastic geometry to derive the transmission probability and success probability for a typical node, and from there two spatial performance metrics, the density of successful transmissions and the spatial fairness index, respectively, were computed. The computations were based on the on approximation of the interference in a CSMA network via a non-homogenous Poisson process. By capturing the delicate interactions among system parameters (qualification threshold $\gamma$, carrier sense threshold $v$ ), the density of transmitters, aggregate interference, and spatial reuse and fairness, we showed that QT-CSMA achieves a higher spatial reuse and fairness than O-CSMA, and more interestingly the simple version of QT-CSMA with one less parameter achieves robust spatial reuse for a wide range of node densities. To better understand the interactions between joint spatial reuse and fairness performance, we characterized the dominated sets of spatial fairness-reuse pair under QT/O-CSMA. Although O-CSMA has one more parameter to adjust, its dominated set was smaller than that of QT-CSMA. Surprisingly, $\mathrm{QT}_{0}$-CSMA which has one less parameter has dominated set which is as large as that of QT-CSMA. This shows that $\mathrm{QT}_{0^{-}}$ CSMA is not only easy to configure and robust but also has high performance.

\section{DERIVATION OF $\tilde{u}\left(n, t_{0}, \tau, \lambda\right)$}

In this appendix, we derive $\tilde{u}$ for $n \in\{0,1,2, \ldots\}, t_{0} \in$ $[0,1), \tau>0$, and $\lambda>0$, which is defined as

$$
\begin{aligned}
\tilde{u}\left(n, t_{0}, \tau, \lambda\right) & =\mathbb{P}\left(E_{1}=1 \mid E_{0}=1, N_{0}=n,\right. \\
T_{0} & \left.=t_{0},\left\{y_{0}, y_{1}\right\} \subset \Phi,\left|y_{0}-y_{1}\right|=\tau\right) .
\end{aligned}
$$

Let $\Phi=\left\{X_{i}\right\}$ be an homogeneous PPP with density $\lambda . N_{0}$ is the number of neighbors of $y_{0}$ and $T_{0}$ is the timer value of $y_{0}$. In the sequel, we omit the conditioning events $\left\{y_{0}, y_{1}\right\} \subset \Phi$ and $\left|y_{0}-y_{1}\right|=\tau$ for simplicity. By applying Bayes' rule, we have

$$
\begin{aligned}
\mathbb{P}\left(E_{1}\right. & \left.=1 \mid E_{0}=1, N_{0}=n, T_{0}=t_{0}\right) \\
& =\frac{\mathbb{P}\left(E_{1}=1, E_{0}=1 \mid N_{0}=n, T_{0}=t_{0}\right)}{\mathbb{P}\left(E_{0}=1 \mid N_{0}=n, T_{0}=t_{0}\right)} .
\end{aligned}
$$


The denominator is simply given by

$$
\mathbb{P}\left(E_{0}=1 \mid N_{0}=n, T_{0}=t_{0}\right)=\left(1-t_{0}\right)^{n}
$$

since for $y_{0}$ to transmit, all its $n$ neighbors should have timer values larger than $t_{0}$ independently. To compute the numerator, we condition on the event that $y_{0}$ and $y_{1}$ are neighbors, i.e., $\left\{y_{1} \in \mathcal{N}_{0}\right\}=\left\{F_{01}^{\prime}>v\left\|y_{0}-y_{1}\right\|^{\alpha}\right\}$, then by the law of total probability we have

$$
\begin{aligned}
\mathbb{P}\left(E_{1}=\right. & \left.E_{0}=1 \mid N_{0}=n, T_{0}=t_{0}\right) \\
= & \underbrace{\mathbb{P}\left(E_{1}=E_{0}=1, y_{1} \in \mathcal{N}_{0} \mid N_{0}=n, T_{0}=t_{0}\right)}_{=0} \\
& +\underbrace{\mathbb{P}\left(E_{1}=E_{0}=1 \mid N_{0}=n, T_{0}=t_{0}, y_{1} \notin \mathcal{N}_{0}\right)}_{c} \\
& \times \underbrace{\mathbb{P}\left(y_{1} \notin \mathcal{N}_{0} \mid N_{0}=n, T_{0}=t_{0}\right) .}_{d}
\end{aligned}
$$

If $y_{0}$ and $y_{1}$ can see each other, it is impossible for both to transmit at the same time, so the first term is equal to 0 . We denote the second and third terms by $c$ and $d$ respectively.

\section{A. Computing $c$ in (48)}

The term $c$ is a conditional probability given on that $y_{0}$ and $y_{1}$ do not see each other. However, they may share some neighbors; so to compute this term we need to further condition on the event that some of $y_{0}$ 's contenders are also seen by $y_{1}$. Let $\mathcal{K}=\mathcal{N}_{0} \cap \mathcal{N}_{1}$ be the set of shared neighbors between $y_{0}$ and $y_{1}$ and $K(\tau) \equiv|\mathcal{K}|$ be the number of them. Note that $K(\tau) \sim \operatorname{Poisson}(\bar{K}(\tau))$ where the mean $\bar{K}(\tau)$ is given by

$$
\begin{aligned}
\bar{K}(\tau) & =\mathbb{E} \sum_{X_{i} \in \Phi} \mathbf{1}\left\{F_{i 0}^{\prime}>v\left\|X_{i}-y_{0}\right\|^{\alpha}\right\} \mathbf{1}\left\{F_{i 1}^{\prime}>v\left\|X_{i}-y_{1}\right\|^{\alpha}\right\} \\
& =\lambda \int_{\mathbb{R}^{2}} \mathbb{P}\left(F_{i 0}>v\|x\|^{\alpha}\right) \mathbb{P}\left(F_{i 1}>v\left\|x-y_{1}\right\|^{\alpha}\right) d x \\
& =\lambda \int_{0}^{2 \pi} \int_{0}^{\infty} e^{-\mu v\left(r^{\alpha}+\left(\tau^{2}+r^{2}-2 \tau r \cos \theta\right)^{\frac{\alpha}{2}}\right)} r d r d \theta .
\end{aligned}
$$

By conditioning on the number of shared neighbors, $K(\tau)$, we rewrite $c$ as

$$
c=\sum_{k=0}^{n} a_{k} b_{k}
$$

where

$a_{k} \equiv \mathbb{P}\left(E_{0}=E_{1}=1 \mid N_{0}=n, T_{0}=t_{0}, K(\tau)=k, y_{1} \notin \mathcal{N}_{0}\right)$, $b_{k} \equiv \mathbb{P}\left(K(\tau)=k \mid N_{0}=n, T_{0}=t_{0}, y_{1} \notin \mathcal{N}_{0}\right)$.

1) Computing $b_{k}: b_{k}$ is the probability that two nodes $y_{0}$ and $y_{1}$ share $k$ common contenders conditioned on $y_{0}$ having $n$ contenders. Since each contender of $y_{0}$ is independently seen by $y_{1}$, the number of shared contenders $K(\tau)$ given $N_{0}=n$ is a Binomial random variable with parameters $n$ and $p_{s}(\tau)$, where $p_{s}(\tau)$ is the probability that one of $y_{0}$ 's neighbors is seen by $y_{1}$. Then, we have

$$
\begin{aligned}
b_{k} & =\mathbb{P}\left(K(\tau)=k \mid N_{0}=n, T_{0}=t_{0}, y_{1} \notin \mathcal{N}_{0}\right) \\
& \stackrel{a}{=} \mathbb{P}\left(K(\tau)=k \mid N_{0}=n, y_{1} \notin \mathcal{N}_{0}\right) \\
& =\left(\begin{array}{l}
n \\
k
\end{array}\right) p_{s}^{k}(\tau)\left(1-p_{s}(\tau)\right)^{n-k},
\end{aligned}
$$

where in $\stackrel{a}{=}$, we used the fact that $\left\{T_{0}=t_{0}\right\}$ is independent of $\{K(\tau)=k\}$, and $p_{s}(\tau)$ is computed as

$$
\begin{aligned}
p_{s}(\tau) & =\mathbb{P}\left(X \in \mathcal{N}_{1} \mid X \in \mathcal{N}_{0}\right) \\
& \stackrel{a}{=} \frac{\mathbb{E}\left[\mathbb{P}\left(X \in \mathcal{N}_{1}, X \in \mathcal{N}_{0} \mid X\right)\right]}{\mathbb{E}\left[\mathbb{P}\left(X \in \mathcal{N}_{0} \mid X\right)\right]} \\
& =\frac{\lambda \int_{\mathbb{R}^{2}} \mathbb{P}\left(F_{0}^{\prime}>v\|x\|^{\alpha}\right) \mathbb{P}\left(F_{1}^{\prime}>v\left\|x-y_{1}\right\|^{\alpha}\right) d x}{\lambda \int_{\mathbb{R}^{2}} \mathbb{P}\left(F>v\|x\|^{\alpha}\right) d x} \\
& =\frac{\bar{K}(\tau)}{\bar{N}_{0}} .
\end{aligned}
$$

In $\stackrel{a}{=}$, we conditioned on the location of $X$ in $\mathbb{R}^{2}$. Using (9), (54) can be rewritten as $p_{s}(\tau)=2-\frac{b(\tau, \lambda)}{\bar{N}_{0}}$.

2) Computing $a_{k}: a_{k}$ can be rewritten as

$$
\begin{aligned}
& a_{k}=\int_{0}^{1} \mathbb{P}\left(E_{1}=E_{0}=1 \mid N_{0}=n,\right. \\
& \left.T_{0}=t_{0}, K(\tau)=k, T_{1}=t_{1}, y_{1} \notin \mathcal{N}_{0}\right) d t_{1}
\end{aligned}
$$

by conditioning on the event that the timer of $y_{1}$ is equal to $t_{1}$, i.e., $\left\{T_{1}=t_{1}\right\}$. Note that either all the shared neighbors in $\mathcal{K}$ have timer values larger than $t_{1}$, i.e., $\left\{T_{j}^{c} \geq t_{1}, \forall C_{j} \in \mathcal{K}\right\}$ where $T_{j}^{c}$ is the timer value of contender $C_{j} \in \mathcal{K}$, or there exist one or more neighbors with timer value(s) smaller than $t_{1}$, i.e., $\left\{\exists C_{j}\right.$ s.t. $\left.T_{j}^{c}<t_{1}\right\}$. Using the law of total probability, the probability inside the integral can be written as (55), (56) and (57) and it is shown at the top of the next page. Let (55), (56), and (57) be $a_{k 1}, a_{k 2}$, and $a_{k 3}$ respectively. We have $a_{k 3}=0$ since if there exists a neighbor with timer value strictly smaller than $t_{1}$, it prevents $y_{1}$ from transmitting, so $E_{1}$ cannot be 1 . $a_{k 2}$ is the probability that all shared neighbors in $\mathcal{K}$ have timer values larger than $t_{1}$, which is simply given as $A_{k 2}=\left(1-t_{1}\right)^{k}$ since each timer is independent and uniform in $[0,1]$. Before we compute $a_{k 1}$, we need to define several random variables.

- Let $N_{1} \sim$ Poisson $\left(\bar{N}_{0}\right)$ be a random variable denoting the number of contenders of $y_{1}$.

- Let $N_{1 x} \sim \operatorname{Poisson}\left(\bar{N}_{1 x}\right)$ be a random variable denoting the number of contenders of $y_{1}$ which are not shared by $y_{0}$. Note that $N_{1 x}+K=N_{1}$ and $\bar{N}_{1 x}=\bar{N}_{0}\left(1-p_{s}\right)$.

- Let $N_{1 x}^{<t_{1}} \sim \operatorname{Poisson}\left(\bar{N}_{1 x}^{<t_{1}}\right)$ be a random variable denoting the number of contenders of $y_{1}$ which are not shared by $y_{0}$ and with timer values smaller than $t_{1}$. Note that $\bar{N}_{1 x}^{<t_{1}}=t_{1} \bar{N}_{1 x}=t_{1} \bar{N}_{0}\left(1-p_{s}\right)$.

To compute $a_{k 1}$, we consider the following two sub-cases $t_{1} \leq$ $t_{0}$ and $t_{0}<t_{1}$. If $t_{1} \leq t_{0}$, then

- $y_{1}$ transmits (or $E_{1}=1$ ) only if it finds no additional neighbors who have timer values smaller than $t_{1}$ and are not seen by $y_{0}$, i.e, if $N_{1 x}^{<t_{1}}=0$, and

- $y_{0}$ transmits (or $E_{0}=1$ ) only if all $T_{j}^{c} \sim \operatorname{Uniform}\left[t_{1}, 1\right]$, $\forall C_{j} \in \mathcal{K}$, are larger than $t_{0}$, which happens with probability $\left(\frac{1-t_{0}}{1-t_{1}}\right)^{k}$ and remaining $n-k$ contenders have timer values larger than $t_{0}$, which happens with probability $\left(1-t_{0}\right)^{n-k}$.

Note that, as in the previous case, $\left\{E_{0}=1\right\}$ and $\left\{E_{1}=0\right\}$ are conditionally independent; so that we have

$$
a_{k 1}=e^{-\bar{N}_{1 x}^{<t_{1}}} \frac{\left(1-t_{0}\right)^{n}}{\left(1-t_{1}\right)^{k}} \quad \text { if } t_{0} \geq t_{1} .
$$




$$
\begin{aligned}
\mathbb{P}\left(E_{1}=\right. & \left.E_{0}=1 \mid N_{0}=n, T_{0}=t_{0}, K=k, T_{1}=t_{1}, y_{1} \notin \mathcal{N}_{0}\right) \\
= & \underbrace{\mathbb{P}\left(E_{1}=E_{0}=1 \mid N_{0}=n, T_{0}=t_{0}, K=k, T_{1}=t_{1}, y_{1} \notin \mathcal{N}_{0}, T_{j}^{c} \geq t_{1} \forall C_{j} \in \mathcal{K}\right)}_{\equiv a_{k 1}} \\
& \times \underbrace{\mathbb{P}\left(T_{j}^{c} \geq t_{1} \forall C_{j} \in \mathcal{K} \mid N_{0}=n, T_{0}=t_{0}, K=k, T_{1}=t_{1}, y_{1} \notin \mathcal{N}_{0}\right)}_{\equiv a_{k 2}=\left(1-t_{1}\right)^{k}} \\
& +\underbrace{\mathbb{P}\left(E_{1}=1, E_{0}=1 \mid N_{0}=n, T_{0}=t_{0}, K=k, T_{1}=t_{1}, y_{1} \notin \mathcal{N}_{0}, \exists C_{j} \text { s.t. } T_{j}^{c}<t_{1}\right)}_{\equiv a_{k}=0} \\
& \times \mathbb{P}\left(\exists C_{j} \text { s.t. } T_{j}^{c}<t_{1} \mid N_{0}=1, T_{0}=t_{0}, K=k, T_{1}=t_{1}, y_{1} \notin \mathcal{N}_{0}\right) .
\end{aligned}
$$

If $t_{0}<t_{1}$

- $y_{0}$ transmits (or $E_{0}=1$ ) if $n-k$ neighbors have timer values larger than $t_{0}$, which happens with probability $\left(1-t_{0}\right)^{n-k}$, and

- $y_{1}$ transmits (or $E_{1}=1$ ) only when it finds no additional neighbors who have timer values smaller than $t_{1}$ and do not see $y_{0}$, i.e., $N_{1 x}^{<t_{1}}=0$.

Note that, as in the previous case, $\left\{E_{0}=1\right\}$ and $\left\{E_{1}=0\right\}$ are conditionally independent; so we have

$$
a_{k 1}=e^{-\bar{N}_{1 x}^{<t_{1}}}\left(1-t_{0}\right)^{n-k} \quad \text { if } t_{0}<t_{1} .
$$

$a_{k 1}$ in the above two cases can be written as follows using indicator functions:

$$
\begin{aligned}
a_{k 1}= & e^{-t_{1} \bar{N}_{0}\left(1-p_{s}\right)}\left(\frac{\left(1-t_{0}\right)^{n}}{\left(1-t_{1}\right)^{k}} \mathbf{1}\left\{t_{1} \leq t_{0}\right\}\right. \\
& \left.+\left(1-t_{0}\right)^{n-k} \mathbf{1}\left\{t_{0}<t_{1}\right\}\right) .
\end{aligned}
$$

Unconditioning with respect to the event $\left\{T_{1}=t_{1}\right\}$ in $a_{k 1} a_{k 2}$ gives (60) and it is shown at the top of the next page where in $\stackrel{a}{=}, \Gamma(a, x)=\int_{x}^{\infty} t^{a-1} e^{-t} d t$ is the incomplete gamma function with $\Gamma(a) \equiv \Gamma(a, 0)$ and $\eta=\bar{N}_{0}\left(1-p_{s}\right)\left(t_{0}-1\right)$. In $\stackrel{b}{=}$ we used the fact that $\frac{\Gamma(k+1, \eta)}{\Gamma(k+1)}=\sum_{j=0}^{k} \frac{\eta^{j}}{j !} e^{-\eta}$. Replacing (60) and (53) in (52) gives

$$
c=\mathbb{P}\left(E_{1}=E_{0}=1 \mid N_{0}=n, T_{0}=t_{0}, y_{1} \notin \mathcal{N}_{0}\right)=\sum_{k=0}^{n} a_{k} b_{k} .
$$

\section{B. Computing $d$ in (49)}

We now compute $d=\mathbb{P}\left(y_{1} \notin \mathcal{N}_{0} \mid N_{0}=n, T_{0}=t_{0}\right)$ in (49). Note that $\left\{N_{0}=n\right\}$ and $\left\{y_{1} \notin \mathcal{N}_{0}\right\}$ are not independent since it is likely that $y_{1}$ is the neighbor of $y_{0}$ if $N_{0}=n$ is large, but $\left\{y_{1} \notin \mathcal{N}_{0}\right\}$ and $\left\{T_{0}=t_{0}\right\}$ are independent since being neighbor of a node does not depend on timer values. Thus, we have $d=\mathbb{P}\left(y_{1} \notin \mathcal{N}_{0} \mid N_{0}=n\right)$. Applying Bayes' rule, we get

$$
\begin{aligned}
d & =\frac{\mathbb{P}\left(y_{1} \notin \mathcal{N}_{0}, N_{0}=n\right)}{\mathbb{P}\left(N_{0}=n\right)} \\
& =\frac{\mathbb{P}\left(N_{0}=n \mid y_{1} \notin \mathcal{N}_{0}\right) \mathbb{P}\left(y_{1} \notin \mathcal{N}_{0}\right)}{\mathbb{P}\left(N_{0}=n\right)},
\end{aligned}
$$

where we have

$$
\mathbb{P}\left(N_{0}=n \mid y_{1} \notin \mathcal{N}_{0}\right)=\frac{\bar{N}_{0}^{n}}{n !} e^{-\bar{N}_{0}}
$$

and

$$
\mathbb{P}\left(y_{1} \notin \mathcal{N}_{0}\right)=\mathbb{P}\left(F_{10}^{\prime}<v \tau^{\alpha}\right)=G\left(v \tau^{\alpha}\right)
$$

for the numerator. To compute the denominator ${ }^{12}$ in (62), namely $\mathbb{P}\left(N_{0}=n\right)$, we need to consider whether $y_{1}$ is seen by $y_{0}$ or not. Using the law of total probability, we have

$$
\begin{aligned}
\mathbb{P}\left(N_{0}=n\right)= & \mathbb{P}\left(N_{0}=n \mid y_{1} \in \mathcal{N}_{0}\right) \mathbb{P}\left(y_{1} \in \mathcal{N}_{0}\right) \\
& +\mathbb{P}\left(N_{0}=n \mid y_{1} \notin \mathcal{N}_{0}\right) \mathbb{P}\left(y_{1} \notin \mathcal{N}_{0}\right) \\
= & \frac{\bar{N}_{0}^{n-1}}{(n-1) !} e^{-\bar{N}_{0}}\left(1-G\left(v \tau^{\alpha}\right)\right)+\frac{\bar{N}_{0}^{n}}{n !} e^{-\bar{N}_{0}} G\left(v \tau^{\alpha}\right) \\
= & \frac{\bar{N}_{0}^{n-1}}{(n-1) !} e^{-\bar{N}_{0}}\left(1+\left(\frac{\bar{N}_{0}}{n}-1\right) G\left(\nu \tau^{\alpha}\right)\right)
\end{aligned}
$$

Note that as expected $\mathbb{P}\left(N_{0}=n\right) \rightarrow \mathbb{P}\left(N_{0}=n \mid y_{1} \notin \mathcal{N}_{0}\right)$ as $\tau \rightarrow \infty$ (or $G(\tau) \rightarrow 1)$, and $\mathbb{P}\left(N_{0}=n\right) \rightarrow \mathbb{P}\left(N_{0}=n \mid y_{1} \in\right.$ $\mathcal{N}_{0}$ ) as $\tau \rightarrow 0$ (or $G(\tau) \rightarrow 0$ ). Then, replacing (63), (64), and (65) in (62) gives the following for $n \geq 0$,

$$
d=\frac{\bar{N}_{0} G\left(\nu \tau^{\alpha}\right)}{n+\left(\bar{N}_{0}-n\right) G\left(\nu \tau^{\alpha}\right)} .
$$

Recall that $d$ is the probability that $y_{1}$ is not the neighbor of $y_{0}$ given $N_{0}=n$. Thus, $d \rightarrow 1(d \rightarrow 0)$ as $\tau \rightarrow \infty(\tau \rightarrow 0)$ makes sense.

\section{Computing $\tilde{u}$}

Now, replacing term $c$ in (61) and $d$ in (66) to (49) gives

$$
\begin{aligned}
\mathbb{P}\left(E_{1}\right. & \left.=E_{0}=1 \mid N_{0}=n, T_{0}=t_{0}\right) \\
& =\frac{\bar{N}_{0} G\left(\nu \tau^{\alpha}\right)}{n+\left(\bar{N}_{0}-n\right) G\left(v \tau^{\alpha}\right)} \sum_{k=0}^{n} a_{k} b_{k} .
\end{aligned}
$$

\footnotetext{
${ }^{12}$ Recall that $\mathbb{P}\left(N_{0}=n\right)$ is indeed $\mathbb{P}\left(N_{0}=n \mid\left\|y_{0}-y_{1}\right\|=\tau\right)$.
} 


$$
\begin{aligned}
a_{k} & =\int_{0}^{1} e^{-t_{1} \bar{N}_{0}\left(1-p_{s}\right)}\left(\left(1-t_{0}\right)^{n} \mathbf{1}\left\{t_{1} \leq t_{0}\right\}+\left(1-t_{0}\right)^{n-k}\left(1-t_{1}\right)^{k} \mathbf{1}\left\{t_{0}<t_{1}\right\}\right) d t_{1} \\
& =\left(1-t_{0}\right)^{n} \int_{0}^{t_{0}} e^{-t_{1} \bar{N}_{0}\left(1-p_{s}\right)} d t_{1}+\left(1-t_{0}\right)^{n-k} \int_{t_{0}}^{1}\left(1-t_{1}\right)^{k} e^{-t_{1} \bar{N}_{0}\left(1-p_{s}\right)} d t_{1} \\
& \stackrel{a}{=}\left(1-t_{0}\right)^{n}\left(\frac{1-e^{-t_{0} \bar{N}_{0}\left(1-p_{s}\right)}}{\bar{N}_{0}\left(1-p_{s}\right)}+\frac{\left(t_{0}-1\right) e^{-\bar{N}_{0}\left(1-p_{s}\right)}(\Gamma(k+1, \eta)-\Gamma(k+1))}{\eta^{k+1}}\right) \\
& \stackrel{b}{=}\left(1-t_{0}\right)^{n}\left(\frac{1-e^{-t_{0} \bar{N}_{0}\left(1-p_{s}\right)}}{\bar{N}_{0}\left(1-p_{s}\right)}+\frac{\left(1-t_{0}\right) e^{-\bar{N}_{0}\left(1-p_{s}\right)} k !}{\eta^{k+1}}\left(1-e^{-\eta} \sum_{j=0}^{k} \frac{\eta^{j}}{j !}\right)\right) .
\end{aligned}
$$

Finally, (46) is given by

$$
\begin{aligned}
\tilde{u}= & \frac{\bar{N}_{0} G\left(\nu \tau^{\alpha}\right)}{n+\left(\bar{N}_{0}-n\right) G\left(v \tau^{\alpha}\right)}\left(\frac{1-e^{-t_{0} \bar{N}_{0}\left(1-p_{s}\right)}}{\bar{N}_{0}\left(1-p_{s}\right)}+\left(1-t_{0}\right)\right. \\
& \times e^{-\bar{N}_{0}\left(1-p_{s}\right)} \sum_{k=0}^{n} \frac{k !}{\eta^{k+1}}\left(1-e^{-\eta} \sum_{j=0}^{k} \frac{\eta^{j}}{j !}\right) \\
& \left.\times\left(\begin{array}{l}
n \\
k
\end{array}\right) p_{s}^{k}\left(1-p_{s}\right)^{n-k}\right),
\end{aligned}
$$

where $p_{s}=p_{s}(\tau)=2-\frac{b(\tau, \lambda)}{\bar{N}_{0}}$ and $\eta=\bar{N}_{0}\left(1-p_{s}\right)\left(t_{0}-1\right)$.

\section{PROOF OF LEMMA 1}

The numerator can be computed using the fact that $N_{s, 0}^{\gamma} \sim$ Poisson $\left(\bar{N}_{s, 0}^{\gamma}\right)$ as follows:

$$
\begin{aligned}
\left(\mathbb{E}^{0}\left[\frac{p_{\gamma}}{N_{s, 0}^{\gamma}+1}\right]\right)^{2} & =\left(p_{\gamma} \sum_{n=0}^{\infty} \frac{1}{n+1} \frac{\left(\bar{N}_{s, 0}^{\gamma}\right)^{n}}{n !} e^{-\bar{N}_{s, 0}^{\gamma}}\right)^{2} \\
& =\left(\frac{p_{\gamma} e^{-\bar{N}_{s, 0}^{\gamma}}}{\bar{N}_{s, 0}^{\gamma}}\left(\sum_{n=0}^{\infty} \frac{\left(\bar{N}_{s, 0}^{\gamma}\right)^{n}}{n !}-1\right)\right)^{2} \\
& =\frac{p_{\gamma}^{2} e^{-2 \bar{N}_{s, 0}^{\gamma}}\left(e^{\bar{N}_{s, 0}^{\gamma}}-1\right)^{2}}{\left(\bar{N}_{s, 0}^{\gamma}\right)^{2}} .
\end{aligned}
$$

The denominator could be computed as follows:

$$
\begin{aligned}
\mathbb{E}^{0}\left[\left(\frac{p_{\gamma}}{N_{s, 0}^{\gamma}+1}\right)^{2}\right] & =p_{\gamma}^{2} \mathbb{E}^{0}\left[\frac{1}{\left(N_{s, 0}^{\gamma}+1\right)^{2}}\right] \\
& =p_{\gamma}^{2} \sum_{n=0}^{\infty} \frac{1}{(n+1)^{2}} \frac{\left(\bar{N}_{s, 0}^{\gamma}\right)^{n}}{n !} e^{-\bar{N}_{s, 0}^{\gamma}} \\
& =\frac{p_{\gamma}^{2} e^{-\bar{N}_{s, 0}^{\gamma}}}{\bar{N}_{s, 0}^{\gamma}}\left(\sum_{n=1}^{\infty} \frac{\left(\bar{N}_{s, 0}^{\gamma}\right)^{n}}{n \cdot n !}\right)
\end{aligned}
$$

Using $\sum_{n=1}^{\infty} \frac{x^{n}}{n \cdot n !}=E i(x)-\log x-\eta[33,8.214 .2$, p. 884] with (67) and (67), we get (39).

\section{REFERENCES}

[1] F. Baccelli, B. Blaszczyszyn, and P. Muhlethaler, "An Aloha protocol for multihop mobile wireless networks," IEEE Trans. Inf. Theory, vol. 52, no. 2, pp. 421-436, Feb. 2006.

[2] S. P. Weber, X. Yang, J. G. Andrews, and G. de Veciana, "Transmission capacity of wireless ad hoc networks with outage constraints," IEEE Trans. Inf. Theory, vol. 51, no. 12, pp. 4091-4102, Dec. 2005.
[3] M. Haenggi, J. G. Andrews, F. Baccelli, O. Dousse, and M. Franceschetti, "Stochastic geometry and random graphs for the analysis and design of wireless networks," IEEE J. Sel. Areas Commun., vol. 27, no. 7, pp. 1029-1046, Sep. 2009.

[4] F. Baccelli and B. Blaszczyszyn, "Stochastic analysis of spatial and opportunistic Aloha," IEEE J. Sel. Areas Commun., vol. 27, no. 7, pp. 1105-1119, Sep. 2009

[5] S. P. Weber, J. G. Andrews, and N. Jindal, "The effect of fading, channel inversion, and threshold scheduling on ad hoc networks," IEEE Trans. Inf. Theory, vol. 53, no. 11, pp. 4127-4149, Nov. 2007.

[6] S. Adireddy and L. Tong, "Exploiting decentralized channel state information for random access," IEEE Trans. Inf. Theory, vol. 51, no. 2, pp. 537-562, Feb. 2005.

[7] X. Qin and R. A. Berry, "Distributed approaches for exploiting multiuser diversity in wireless networks," IEEE Trans. Inf. Theory, vol. 52, no. 2, pp. 392-413, Feb. 2006.

[8] H. Q. Nguyen, F. Baccelli, and D. Kofman, "A stochastic geometry analysis of dense IEEE 802.11 networks," in Proc. 26th IEEE INFOCOM, May 2007, pp. 1199-1207.

[9] F. Baccelli and B. Blaszczyszyn, Stochastic Geometry and Wireless Networks, vol. 2. Norwell, MA, USA: Now Publishers, 2009.

[10] S. Patil and G. de Veciana, "Measurement-based opportunistic scheduling for heterogenous wireless systems," IEEE Trans. Commun., vol. 57, no. 9, pp. 2745-2753, Sep. 2009.

[11] S. Patil and G. de Veciana, "Managing resources and quality of service in wireless systems exploiting opportunism," IEEE/ACM Trans. Netw., vol. 15, no. 5, pp. 1046-1058, Oct. 2007.

[12] D. Park, H. Kwon, and B. Lee, "Wireless packet scheduling based on the cumulative distribution function of user transmission rates," IEEE Trans. Commun., vol. 53, no. 11, pp. 1919-1929, Nov. 2005.

[13] T. Bonald, "A score-based opportunistic scheduler for fading radio channels," in Proc. Eur. Wireless, 2004.

[14] C. Hwang and J. M. Cioffi, "Using opportunistic CSMA/CA to achieve multi-user diversity in wireless LAN," in Proc. IEEE Global Telecommun. Conf., Nov. 2007, pp. 4952-4956.

[15] J. Choi and S. Bahk, "Channel aware MAC scheme based on CSMA/CA," in Proc. IEEE 59th VTC, May 2004, pp. 1559-1563.

[16] Y. Kim, "Spatial spectrum reuse in wireless networks: Design and performance," Ph.D. dissertation, Dept. Elect. Comput. Eng., Univ. Texas at Austin, Austin, TX, USA, 2011.

[17] H. Chhaya and S. Gupta, "Performance modeling of asynchronous data transfer methods of IEEE 802.11 MAC protocol," Wireless Netw., vol. 3, no. 3, pp. 217-234, 1997

[18] M. M. Carvalho and J. J. Garcia-Luna-Aceves, "A scalable model for channel access protocols in multihop ad hoc networks," in Proc. 10th Annu. Int. Conf. Mobile Computing and Netw., New York, NY, USA 2004, pp. 330-344.

[19] D. Stoyan, W. S. Kendall, and J. Mecke, Stochastic Geometry and Its Applications. New York, NY, USA: Wiley, 1995.

[20] R. Giacomelli, R. K. Ganti, and M. Haenggi, "Outage probability of general ad hoc networks in the high-reliability regime," IEEE/ACM Trans. Netw., vol. 19, no. 4, pp. 1151-1163, Aug. 2011.

[21] R. K. Ganti, J. G. Andrews, and M. Haenggi, "High-SIR transmission capacity of wireless networks with general fading and node distribution," IEEE Trans. Inf. Theory, vol. 57, no. 5, pp. 3100-3116, May 2011.

[22] Y. Kim and G. de Veciana, "Joint network capacity region of cognitive networks heterogeneous environments and RF-environment awareness," IEEE J. Sel. Areas Commun., vol. 29, no. 2, pp. 407-420, Feb. 2011 
[23] Y. Kim and G. de Veciana, "Understanding the design space for cognitive networks," in Proc. 6th Workshop Spatial Stochastic Models Wireless Netw., Jun. 2010.

[24] T. V. Nguyen and F. Baccelli, "A probabilistic model of carrier sensing based cognitive radio," in Proc. IEEE Symp. New Frontiers Dynamic Spectrum, Apr. 2010, pp. 1-12.

[25] F. Baccelli and B. Blaszczyszyn, Stochastic Geometry and Wireless Networks, vol. 1. Norwell, MA, USA: Now Publishers, 2009.

[26] P. Brémaud, Mathematical Principles of Signal Processing. New York, NY, USA: Springer-Verlag, 2002.

[27] M. Durvy, O. Dousse, and P. Thiran, "On the fairness of large CSMA networks," IEEE J. Sel. Areas Commun., vol. 27, no. 7, pp. 1093-1104, Sep. 2009.

[28] X. Wang and K. Kar, "Throughput modelling and fairness issues in CSMA/CA based ad-hoc networks," in Proc. 24th Annu. Joint Conf. IEEE INFOCOM, vol. 1. Mar. 2005, pp. 23-34.

[29] M. Durvy, O. Dousse, and P. Thiran, "Self-organization properties of CSMA/CA systems and their consequences on fairness," IEEE Trans. Inf. Theory, vol. 55, no. 3, pp. 931-943, Mar. 2009.

[30] P. M. van de Ven, J. S. H. van Leeuwaarden, D. Denteneer, and A. J. E. M. Janssen, "Spatial fairness in linear random-access networks," Perform. Eval., vol. 69, nos. 3-4, pp. 121-134, 2010.

[31] P. M. van de Ven, S. C. Borst, D. Denteneer, A. J. E. M. Janssen, and J. S. H. van Leeuwaarden, "Equalizing throughputs in random-access networks," SIGMETRICS Perform. Eval. Rev., vol. 38, no. 2, pp. 39-41, Oct. 2010.

[32] R. K. Jain, D. M. Chiu, and W. R. Hawe, "A quantitative measure of fairness and discrimination for resource allocation in shared computer systems," Digital Equipment Corporation, Hudson, MA, USA, Tech. Rep. TR-301, Sep. 1984.

[33] I. S. Gradshteyn and I. M. Ryzhik, Tables of Integrals, Series, and Products, 7th ed. New York, NY, USA: Academic, 2007.

Yuchul Kim received his B.S. degree from Korea University in 2000 and M.S. degree from Korea Advanced Institute of Science and Technology in 2003, and Ph.D. from The University of Texas at Austin, all in Electrical Engineering. He had worked in Global Standardization and Research Lab in the Telecommunication and Network division of Samsung Electronics from 2003 to 2006. His research includes the design, modeling and performance evaluation of wireless ad-hoc and cellular networks.
François Baccelli is Simons Math $+X$ Chair in Mathematics and ECE at UT Austin. His research directions are at the interface of Applied Mathematics (Probability, Stochastic Geometry, Dynamical Systems) and Communications (Network Science, Information Theory). He is co-author of research monographs on point processes and queues (with P. Brémaud); max plus algebras and network dynamics (with G. Cohen, G. Olsder and J. P. Quadrat); stationary queuing networks (with P. Brémaud); stochastic geometry and wireless networks (with B. Blaszczyszyn). He is a member of the French Academy of Sciences and part time researcher at INRIA.

Gustavo de Veciana (S'88-M'94-SM'01-F'09) received his B.S., M.S, and $\mathrm{Ph} . \mathrm{D}$. in electrical engineering from the University of California at Berkeley in 1987, 1990, and 1993 respectively. He is currently the Joe. J. King Professor at the Department of Electrical and Computer Engineering. He served as the Director and Associate Director of the Wireless Networking and Communications Group (WNCG) at the University of Texas at Austin, from 2003-2007. His research focuses on the analysis and design of wireless and wireline telecommunication networks; architectures and protocols to support sensing and pervasive computing; applied probability and queuing theory. Dr. de Veciana has served as editor for the IEEE/ACM TRANSACTIONS ON NETWORKING. He was the recipient of a National Science Foundation CAREER Award 1996, co-recipient of the IEEE William McCalla Best ICCAD Paper Award for 2000, co-recipient of the Best Paper in ACM Transactions on Design Automation of Electronic Systems, Jan 2002-2004, co-recipient of the Best Paper in the International Teletraffic Congress (ITC- 22) 2010, and of the Best Paper in ACM International Conference on Modeling, Analysis and Simulation of Wireless and Mobile Systems 2010. He is on the technical advisory board of IMDEA Networks. 